\title{
Orbits of 152 Globular Clusters of the Milky Way Galaxy Constructed from the Gaia DR2 data
}

\author{
A. T. Bajkova, V. V. Bobylev \\ Pulkovo Astronomical Observatory, St.-Petersburg, Russia, E-mail: bajkova@gaoran.ru
}

\begin{abstract}
We present orbits and their properties for 152 globular clusters of the Milky Way galaxy obtained using average Gaia DR2 proper motions and other astrometric data from the list of Vasiliev (2019). For orbit integrating we have used the axisymmetric model of the Galactic potential based on the Navarro-Frenk-White dark halo, and modified by Bajkova, Bobylev (2016, 2017) using circular velocities of Galactic objects in wide region of Galactocentric distances (up to $200 \mathrm{kpc}$ ) from Bhattacharjee et al. (2014) catalog. Based on the analysis of the obtained orbits, we have modified the composition of the subsystems of globular clusters presented in Massari et al. (2019).
\end{abstract}

Key words: (Galaxy:) globular clusters: general

\section{Introduction}

The appearance of accurate astrometric data from measurements from the Gaia satellite of the positions and spatial velocities of globular clusters (Helmi et al. 2018; Baumgardt et al. 2019; Vasiliev 2019) makes it possible to study their dynamics, origin and evolution (Myeong et al. 2019; Massari et al. 2019; Bajkova et al. 2020).

In this work we present orbits and their properties for the almost entire list of globular clusters compiled by Vasiliev (2019) on the basis of the most accurate measurements of their velocities and positions to date and using one of the best-fit models of the Milky Way gravitational potential. In addition, we set a goal to revise the classification of globular clusters proposed by Massari et al. (2019) on the basis of analysis of the obtained orbits. In essence, this paper is a supplement to paper of Bajkova et al. (2020), which was devoted to the division of globular clusters into subsystems of the Galaxy, namely, bulge/bar, thick disk, and halo. Recall that in paper of Bajkova et al. (2020) a new criterion of separation of globular clusters belonging to the disk and halo of the Galaxy was proposed. This criterion is based on the bimodality of the GCs distribution $L_{Z} / e c c$, where $L_{Z}$ is $Z$ component of the angular momentum, ecc is eccentricity of the orbit.

In addition to work of Bajkova et al. (2020), we present here a broader set of orbital parameters, and most importantly, we present a catalog of orbit images in two projections, which makes it possible to more fully analyze them due to visualization. Such work on visualization of the orbits of almost all globular clusters known to date, and carried out according to the latest data, has been done in the literature for the first time. This work provides, in addition to the available quantitative estimates, the possibility of a qualitative assessment of the GC dynamics, comparison of the orbits of different GCs included in different classification groups.

In the previous work, we dealt only with the problem of dividing the GCs into formed in situ and clusters which formed in different progenitors that were only later accreted, i.e. division into disk, bulge and halo subsystems. We did not touch upon the problem of classifying the halo GCs into subsystems, the nature of which is the accretion events of members of other galaxies (the Sausage, Sequoia) onto the Milky Way. An attempt at such a classification was made, for example, in the work 
of Massari et al. (2019). But we do not consider this work completed, since the analysis of the various diagrams suggests some inconsistencies and contradictions. A qualitative analysis of the orbits allowed us to make some adjustments to the Massari's classification. As a result, we offer our own modified classification, which seems to be more organic. The change in classification affected 27 objects.

In addition to the previous article, we present the values of important orbital parameters that were not considered earlier.

This work is structured as follows. Section 2 describes the accepted most realistic model for the axially symmetric Galactic potential. Section 3 devoted to integrating the orbits and computing parameters of the orbits. Section 4 describes data. In Section 5 we present orbits of 152 globular clusters and their properties and propose a modified classification of the GCs based on the analysis of orbits which is slightly different from the classification given by Massari et al. (2019). In Conclusions we summarize main results.

\section{Model for the Axially Symmetric Galactic Potential}

In this article the same model of gravitational potential as in work by Bajkova et al. (2020) is adopted. The axially symmetric gravitational potential of the Galaxy is represented as the sum of three components - the central, spherical bulge $\Phi_{b}(r(R, Z))$, the disk $\Phi_{d}(r(R, Z))$, and the massive, spherical dark-matter halo $\Phi_{h}(r(R, Z))$ :

$$
\Phi(R, Z)=\Phi_{b}(r(R, Z))+\Phi_{d}(r(R, Z))+\Phi_{h}(r(R, Z)) .
$$

Here, we use a cylindrical coordinate system $(R, \psi, Z)$ with its origin at the Galactic center. In Cartesian coordinates $(X, Y, Z)$ with their origin at the Galactic center, the distance to a star (the spherical radius) is $r^{2}=X^{2}+Y^{2}+Z^{2}=R^{2}+Z^{2}$. The gravitational potential is expressed in units of $100 \mathrm{~km}^{2} \mathrm{~s}^{-2}$, distances in kpc, masses in units of the mass of the Galaxy, $M_{\text {gal }}=2.325 \times 10^{7} M_{\odot}$, and the gravitational constant is taken to be $G=1$.

We express the potentials of the bulge, $\Phi_{b}(r(R, Z))$, and disk, $\Phi_{d}(r(R, Z))$, in the form suggested by Miyamoto, Nagai (1975):

$$
\begin{gathered}
\Phi_{b}(r)=-\frac{M_{b}}{\left(r^{2}+b_{b}^{2}\right)^{1 / 2}}, \\
\Phi_{d}(R, Z)=-\frac{M_{d}}{\left[R^{2}+\left(a_{d}+\sqrt{Z^{2}+b_{d}^{2}}\right)^{2}\right]^{1 / 2}},
\end{gathered}
$$

where $M_{b}, M_{d}$ are the masses of these components, and $b_{b}, a_{d}, b_{d}$ are the scale parameters of the components in kpc.

For description of the halo component, we used the expression in Navarro-Frenk-White (NFW) form presented in Navarro et al. (1997):

$$
\Phi_{h}(r)=-\frac{M_{h}}{r} \ln \left(1+\frac{r}{a_{h}}\right),
$$

where $M_{h}$ is the mass, $a_{h}$ is the scale length.

The model of the Galactic potential, considered in this work, is the NFW model modified in work of Bajkova \& Bobylev (2016) by fitting of the model parameters to data on HI, maser sources and Galactic objects from Bhattacharjee et al. (2014) at distances $R$ within $\sim 200 \mathrm{kpc}$. In addition the constraints (Irrgang et al. 2013) on the local dynamical matter density $\rho_{\odot}=0.1 M_{\odot} \mathrm{pc}^{-3}$ and the force acting perpendicularly to the Galactic plane $\left|K_{z=1.1}\right| / 2 \pi G=77 M_{\odot} \mathrm{pc}^{-2}$ were used. 
Note that among six models of the Galactic potential summarized in Bajkova \& Bobylev (2017) our model (denoted as Model III in papers of Bajkova \& Bobylev (2016, 2017) ensures the best fit to the data. Here we denote the model as NFWBB for short.

Parameters of the NFWBB model are given in Table 1 of work of Bajkova et al. (2020). Corresponding rotation curves up to $R=200 \mathrm{kpc}$ are shown in Fig.3 of work by Bajkova \& Bobylev (2016). When deriving the model rotation curve, we used $R_{\odot}=8.3 \mathrm{kpc}$ for the Galactocentric distance of the Sun and $V_{\odot}=244 \mathrm{~km} \mathrm{~s}^{-1}$ for the linear velocity of the Local Standard of Rest around the center of the Galaxy. The mass of the Galaxy according to this model is $M_{G_{(R \leq 200 k p c)}}=0.75 \pm 0.19 \times 10^{12} M_{\odot}$. This value is consistent with the recently obtained estimate of the lower mass limit for the dark spherical NFW halo $M_{200}=0.67_{-0.15}^{+0.30} \times 10^{12} M_{\odot}($ Koppelman \& Helmi, 2020) from the escape velocity using a proper motion selected halo sample.

\section{Integrating the Orbits and Computing Orbit Parameters}

The equation of motion of a test particle in an axially symmetric gravitational potential can be obtained from the Lagrangian of the system $£$ (see Appendix A in Irrgang et al. (2013)):

$$
\begin{aligned}
& £(R, Z, \dot{R}, \dot{\psi}, \dot{Z})= \\
& \quad 0.5\left(\dot{R}^{2}+(R \dot{\psi})^{2}+\dot{Z}^{2}\right)-\Phi(R, Z) .
\end{aligned}
$$

Introducing the canonical moments

$$
\begin{aligned}
& p_{R}=\partial £ / \partial \dot{R}=\dot{R} \\
& p_{\psi}=\partial £ / \partial \dot{\phi}=R^{2} \dot{\psi} \\
& p_{Z}=\partial £ / \partial \dot{Z}=\dot{Z}
\end{aligned}
$$

we obtain the Lagrangian equations in the form of a system of six first-order differential equations:

$$
\begin{aligned}
& \dot{R}=p_{R}, \\
& \dot{\psi}=p_{\psi} / R^{2}, \\
& \dot{Z}=p_{Z}, \\
& \dot{p_{R}}=-\partial \Phi(R, Z) / \partial R+p_{\psi}^{2} / R^{3}, \\
& \dot{p_{\psi}}=0, \\
& \dot{p_{Z}}=-\partial \Phi(R, Z) / \partial Z .
\end{aligned}
$$

We integrated Eqs. (7) using a fourth-order Runge-Kutta algorithm.

The Sun's peculiar velocity with respect to the Local Standard of Rest was taken to be $\left(u_{\odot}, v_{\odot}, w_{\odot}\right)=(11.1,12.2,7.3) \pm(0.7,0.5,0.4) \mathrm{km} \mathrm{s}^{-1}(($ Schonrich et al. 2010)). Here, we use the heliocentric velocities in a moving Cartesian coordinate system with $u$ directed towards the Galactic center, $v$ in the direction of the Galactic rotation, and $w$ perpendicular to the Galactic plane and directed towards the north Galactic pole.

Let the initial positions and space velocities of a test particle in the heliocentric coordinate system be $\left(x_{o}, y_{o}, z_{o}, u_{o}, v_{o}, w_{o}\right)$. The initial positions $(X, Y, Z)$ and velocities $(U, V, W)$ of the test particle in Galactic Cartesian coordinates are then given by the formulas:

$$
\begin{aligned}
& X=R_{\odot}-x_{o}, Y=y_{o}, Z=z_{o}+h_{\odot}, \\
& R=\sqrt{X^{2}+Y^{2}}, \\
& U=u_{o}+u_{\odot}, \\
& V=v_{o}+v_{\odot}+V_{\odot}, \\
& W=w_{o}+w_{\odot},
\end{aligned}
$$


where $R_{\odot}$ and $V_{\odot}$ are the Galactocentric distance and the linear velocity of the Local Standard of Rest around the Galactic center, $h_{\odot}=16$ pc (Bajkova \& Bobylev, 2016) is the height of the Sun above the Galactic plane, $\Pi$ and $\Theta$ are radial and tangential (rotational) velocities respectively.

Below we present the following orbital parameters of globular clusters:

(1)initial distance of the GC from the Galactic center $d_{G C}$ :

$$
d_{G C}=\sqrt{X^{2}+Y^{2}+Z^{2}}
$$

(2)radial velocity $\Pi$ :

$$
\Pi=-U \frac{X}{R}+V \frac{Y}{R}
$$

(3)tangential velocity $\Theta$ :

$$
\Theta=U \frac{Y}{R}+V \frac{X}{R}
$$

(4)total $3 \mathrm{D}$ velocity $V_{t o t}$ :

$$
V_{t o t}=\sqrt{\Pi^{2}+\Theta^{2}+W^{2}} ;
$$

(5)apocentric distance (apo) of the orbit;

(6)pericentric distance (peri) of the orbit;

(7)the eccentricity (ecc) of the orbit:

$$
e c c=\frac{a p o-p e r i}{a p o+p e r i}
$$

(8)the components of the angular momentum:

$$
\begin{aligned}
& L_{X}=Y \times W-Z \times V ; \\
& L_{Y}=Z \times U-X \times W ; \\
& L_{Z}=X \times V-Y \times U ;
\end{aligned}
$$

(9)inclination of the orbit $\theta$ :

$$
\theta=\arccos \left(\frac{L_{Z}}{L}\right)
$$

where $L=\sqrt{L_{X}^{2}+L_{Y}^{2}+L_{Z}^{2}}$;

(10)period of the orbit $T_{r}$;

(11)total energy $E$ :

$$
E=\Phi(R, Z)+\frac{V_{t o t}^{2}}{2} .
$$

\section{Data}

In this paper, as the source of data on globular clusters the Vasiliev (2019) catalog serves. It contains average proper motions calculated from data of the Gaia DR2 Catalog, line-of-sight velocities, $(\alpha, \delta)$ positions and distances of 150 globular clusters. Data for GC Liller 1 we took from previous preprint of Vasiliev (2018). For the globular cluster FSR 1758, we took data from the work of Villanova et al. (2019).

The initial position and velocity coordinates (the $6 \mathrm{~d}$ phase space) $\left(x_{o}, y_{o}, z_{o}, u_{o}, v_{o}, w_{o}\right)$ were calculated from these data and used for integrating the orbits. Uncertainties in the initial coordinates were 
calculated using Monte-Carlo simulation (1000 iterations) taking into account the measurement errors given in catalog of Vasiliev (2019). We adopted the uncertainty in the GCs heliocentric distances $d$ as $7 \%$, which is about 1.5 times larger than the estimate given by Vasiliev (2019).

Actually, Vasiliev (2019) used the distances from the Harris (2010) catalog, where it is assumed an error of 0.1 in distance modulus, corresponding to a relative error of $4.6 \%$ in the distance, which, according to Vasiliev (2019), is a rather optimistic choice, since for some clusters, as follows from independent literature sources, the variation in several independent distance estimates could exceed 0.1 mag. It should be borne in mind that there is a possibility of additional error due to inhomogeneity in absorption in the Galaxy, but this is unlikely to give a contribution greater than 0.1 mag (color excess error $E(B-V) \sim 0.03$ ). Hence, the total error (square root of the sum of squares) in the distance modulus is hardly more than $0.15 \mathrm{mag}$, which corresponds to about $7 \%$ uncertainty in the distances.

Even in the case of an estimate of $4.6 \%$, the distance seems to be the largest source of uncertainty for most of the clusters (Vasiliev (2019)), the more this is aggravated in our case, when the uncertainty in the distances is assumed to be about 1.5 times larger (7\%). Therefore, it makes sense to study the effect of distance uncertainties on the values of the clusters orbit parameters. Comparison of the most important orbit parameters $\left(E, L_{Z}, e c c, L_{Z} / e c c, V_{t o t}, A p o, \theta, T_{r}\right)$ is given in Fig. 1. In this Figure the parameters obtained for the GCs with the distances which are both larger on $7 \%$ and less on $7 \%$ than the nominal ones, are compared with the corresponding orbit parameters obtained for the GCs with nominal distances.

It should be especially noted that among the analyzed parameters we also included the ratio $L_{Z} /$ ecc, the bimodality of the distribution of which we used in work of Bajkova et al. (2020) to separate globular clusters belonging to the thick disk and halo. The influence of the uncertainty in the GC distances of $7 \%$ led to a standard deviation of this ratio of $5 \%$ (relative to effective range of this parameter), which we expect did not affect the result of GCs classification due to the high stability of the separation algorithm shown in work of Bajkova et al. (2020). The greatest influence from uncertainty in distances of $7 \%$ is experienced by such parameters as eccentricity (ecc) and inclination of orbit $\theta$. The standard deviations of these parameters are 7.6 and $8.4 \%$, respectively. All other parameters are significantly less influenced, with a standard deviation of not larger than $3 \%$ in each case.

\section{Orbits of 152 Globular Clusters and their Properties}

The orbits of the 152 globular clusters were obtained by integrating Eg. (7) for 5 Gyr backward. The $(\mathrm{X}, \mathrm{Y})$ and $(\mathrm{R}, \mathrm{Z})$ orbit projections for each of all GCs are presented in Fig. 2, The orbit properties are presented in Table 1. To calculate the uncertainties in the orbit properties, we used the Monte Carlo method with 100 realizations, taking into account the uncertainties in the initial coordinates and velocities of GCs, as well as errors in the peculiar velocity of the Sun. For parameters $T_{r}, L_{Z}$ and $E$, we give in Table 1 only nominal values to save space.

Based on the analysis of orbits and their properties, a small regrouping of globular clusters by subsystems has been made. Table 2 shows 27 GCs, which have changed their belonging to one group or another. In this table, the column designated as GS - Galactic Subsystem, gives the classification proposed by Massari et al. (2019), and the column designated as GS(m), gives a modified classification. The following designations for the Galactic subsystems are used here: D (disk), B (bulge), GE (GaiaEnceladus, or Gaia-Sausage), H99 (Helmi Streams), Seq (Sequoia galaxy), Sgr (Sagittarius dwarf), HE (unassociated High-Energy), LE (unassociated Low-Energy), XXX (clusters with no available kinematics). 

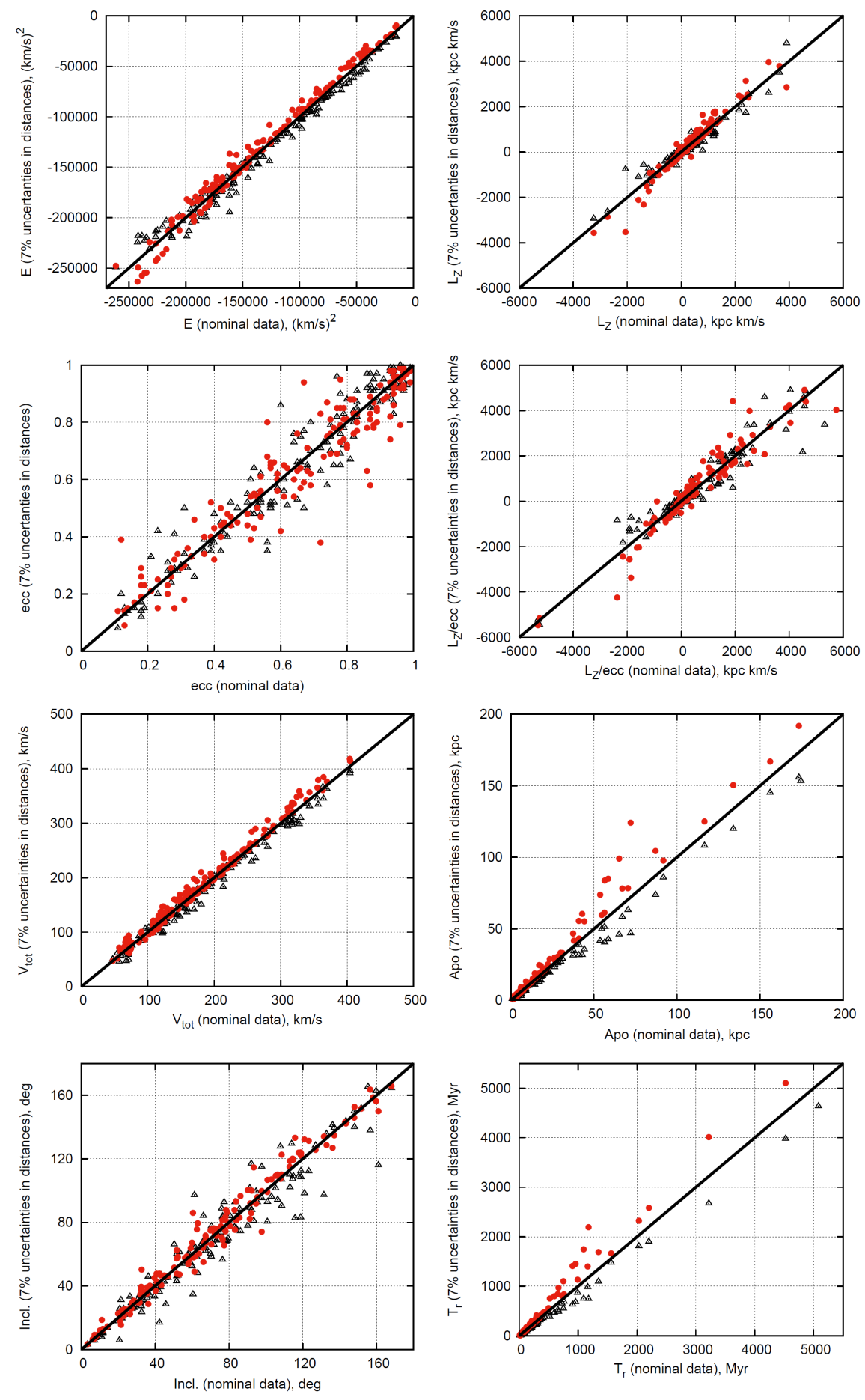

Figure 1: Comparison of the GCs orbit parameters $\left(E, L_{Z}, e c c, L_{Z} / e c c, V_{t o t}, A p o, \theta, T_{r}\right)$. The parameters obtained for the GCs with the distances which are larger on $7 \%$ (red points) and less on $7 \%$ (black triangles) than the nominal ones, are compared with the corresponding orbit parameters obtained for the GCs with nominal distances. In each panel we plot the line of coincidence. 
Table 1: Orbital properties of the GCs. For each GC we quote values derived from orbits integrated for 5 Gyr backward.

\begin{tabular}{|c|c|c|c|c|c|c|c|c|c|c|c|}
\hline Name & $\begin{array}{c}d_{G C} \\
{[\mathrm{kpc}]}\end{array}$ & $\begin{array}{r}\Pi \\
{[\mathrm{km} / \mathrm{s}]}\end{array}$ & $\begin{array}{r}\Theta \\
{[\mathrm{km} / \mathrm{s}]}\end{array}$ & $\begin{array}{r}V_{t o t} \\
{[\mathrm{~km} / \mathrm{s}]}\end{array}$ & $\begin{array}{r}\text { apo } \\
{[\mathrm{kpc}]}\end{array}$ & $\begin{array}{r}\text { peri } \\
{[\mathrm{kpc}]}\end{array}$ & ecc & $\begin{array}{r}\text { incl. } \theta \\
\text { [deg] }\end{array}$ & $\begin{array}{r}T_{r} \\
{[\mathrm{Myr}]}\end{array}$ & $\begin{array}{r}L_{Z} \\
{[\mathrm{kpc}} \\
\mathrm{km} / \mathrm{s}] \\
\end{array}$ & $\begin{array}{r}E \\
{\left[\mathrm{~km}^{2}\right.} \\
\left./ \mathrm{s}^{2}\right] \\
\end{array}$ \\
\hline NGC 104 & 7.6 & $6_{-9}^{+5}$ & $192_{-4}^{+8}$ & $197_{-3}^{+7}$ & $7.7_{-0.1}^{+0.1}$ & $5.5_{-0.2}^{+0.3}$ & $0.16_{-0.03}^{+0.02}$ & $27_{-1}^{+2}$ & 116 & 1328 & -126288 \\
\hline NGC 288 & 12.2 & $\begin{array}{r}+3 \\
-4\end{array}$ & $-42_{-22}^{+28}$ & $66_{-14}^{+16}$ & $12.3_{-0.4}^{+0.3}$ & $1.4_{-0.8}^{+0.7}$ & $0.80_{-0.10}^{+0.11}$ & $119_{-16}^{+\overline{7}}$ & 142 & -349 & -116280 \\
\hline NGC 362 & 9.5 & $127_{-13}^{+18}$ & $-1_{-12}^{+12}$ & $145_{-9}^{+16}$ & $12.1_{-0.6}^{+0.3}$ & $0.1_{-0.0}^{+0.3}$ & $0.99_{-0.06}^{+0.00}$ & $92_{-19}^{+22}$ & 126 & -10 & -121290 \\
\hline Whiting 1 & 34.7 & $-208_{-14}^{+18}$ & $109_{-16}^{+22}$ & $235_{-17}^{+18}$ & $67.0_{-9.0}^{+14.3}$ & $20.3_{-1.3}^{+3.4}$ & $0.54_{-0.05}^{+0.02}$ & $67_{-2}^{+3}$ & 1162 & 2494 & -42265 \\
\hline NGC 1261 & 18.2 & $\begin{array}{l}-95_{-9}^{+11} \\
\end{array}$ & $-19_{-9}^{+10}$ & $119_{-8}^{+9}$ & $21.1_{-1.0}^{+1.4}$ & $0.7_{-0.2}^{+0.4}$ & $0.94_{-0.04}^{+0.02}$ & $121_{-16}^{+11}$ & 244 & -249 & -91385 \\
\hline Pal 1 & 17.4 & $42_{-8}^{+6}$ & $214_{-4}^{+3}$ & $219_{-3}^{+3}$ & $19.1_{-0.9}^{+0.4}$ & $14.6_{-0.8}^{+0.6}$ & $0.13_{-0.02}^{+0.03}$ & $14_{-1}^{+1}$ & 350 & 3646 & -78086 \\
\hline E 1 & 124.7 & $\begin{array}{r}11+75 \\
-72\end{array}$ & $-144_{-88}^{+114}$ & $174_{-45}^{+104}$ & $237.9_{-42.9}^{+45.2}$ & $104.3_{-45.9}^{+30.3}$ & $0.39_{-0.00}^{+0.41}$ & $127_{-28}^{+6}$ & 5594 & -12100 & -15914 \\
\hline Eridanus & 95.2 & $\begin{array}{l}-90_{-31}^{+27} \\
\end{array}$ & $-28_{-27}^{+48}$ & $163_{-16}^{+43}$ & $174.5_{-24.3}^{+83.6}$ & $12.0_{-6.2}^{+73.4}$ & $0.87_{-0.31}^{+0.02}$ & $113_{-37}^{+13}$ & 3220 & -2064 & -23988 \\
\hline Pal 2 & 35.3 & $-108_{-5}^{+5}$ & $11_{-23}^{+22}$ & $108_{-2}^{+8}$ & $41.0_{-2.2}^{+2.0}$ & $0.6_{-0.4}^{+1.8}$ & $0.97_{-0.08}^{+0.02}$ & $42_{-35}^{+70}$ & 506 & 373 & -63896 \\
\hline NGC 1851 & 16.9 & $105_{-4}^{+4}$ & $-1_{-5}^{+5}$ & $133_{-4}^{+4}$ & $20.1_{-0.2}^{+0.7}$ & $0.1_{-0.0}^{+0.2}$ & $0.99_{-0.02}^{+0.00}$ & $92_{-9}^{+9}$ & 232 & -22 & -94063 \\
\hline NGC 1904 & 19.0 & $46_{-3}^{+8}$ & $12_{-9}^{+12}$ & $47_{-0}^{+9}$ & $19.7_{-0.9}^{+0.5}$ & $0.3_{-0.1}^{+0.5}$ & $0.97_{-0.05}^{+0.01}$ & $60_{-25}^{+27}$ & 218 & 211 & -96192 \\
\hline NGC 2298 & 16.0 & $-92_{-8}^{+6}$ & $-32_{-10}^{+8}$ & $125_{-8}^{+7}$ & $18.0_{-0.4}^{+0.7}$ & $1.2_{-0.4}^{+0.5}$ & $0.87_{-0.05}^{+0.04}$ & $118_{-5}^{+8}$ & 208 & -500 & -98716 \\
\hline NGC 2419 & 90.2 & $-5_{-13}^{+10}$ & $47_{-17}^{+30}$ & $75_{-14}^{+31}$ & $91.8_{-6.1}^{+7.3}$ & $17.3_{-5.5}^{+13.3}$ & $0.68_{-0.18}^{+0.09}$ & $52_{-18}^{+14}$ & 1562 & 3907 & -35896 \\
\hline Pyxis & 41.5 & $-247_{-5}^{+9}$ & $\begin{array}{l}-29_{-10}^{+2 i} \\
-10\end{array}$ & $311_{-10}^{+13}$ & $324.2_{-90.9}^{+95.8}$ & $41.7_{-12.8}^{+4.7}$ & $0.77_{-0.01}^{+0.07}$ & $98_{-6}^{+2}$ & 7100 & -1205 & -15098 \\
\hline E 3 & 9.3 & $44_{-16}^{+11}$ & $251_{-18}^{+13}$ & $276_{-18}^{+14}$ & $13.1_{-2.0}^{+1.9}$ & $9.2_{-0.5}^{+0.4}$ & $0.18_{-0.07}^{+0.06}$ & $29_{-1}^{+1}$ & 224 & 2240 & -97684 \\
\hline Pal 3 & 95.9 & $-147_{-70}^{+47}$ & $\begin{array}{r}89_{-62}^{+79} \\
\end{array}$ & $184_{-20}^{+81}$ & $173.4_{-73.9}^{+77.4}$ & $76.3_{-13.7}^{+29.1}$ & $0.39_{-0.00}^{+0.38}$ & $67_{-14}^{+15}$ & 4524 & 6495 & -20091 \\
\hline NGC 3201 & 9.1 & $-114_{-18}^{+17}$ & $-301_{-9}^{+9}$ & $355_{-11}^{+12}$ & $26.3_{-3.0}^{+3.7}$ & $8.4_{-0.3}^{+0.3}$ & $0.52_{-0.05}^{+0.05}$ & $152_{-1}^{+1}$ & 372 & -2728 & -75483 \\
\hline Pal 4 & 111.4 & $-25_{-67}^{+46}$ & $-33_{-60}^{+63}$ & $70_{-0}^{+7 \frac{2}{2}}$ & $116.5_{-8.5}^{+22.1}$ & $16.3_{-1.6}^{+60.0}$ & $0.75_{-0.49}^{+0.01}$ & $103_{-17}^{+7}$ & 2032 & -1394 & -31065 \\
\hline Crater & 144.8 & $-101_{-98}^{+110}$ & $\begin{array}{l}-63_{-270}^{+105} \\
-\end{array}$ & $135_{-0}^{+249}$ & $156.1_{-90.0}^{+97.5}$ & $117.4_{-18.0}^{+42.6}$ & $0.14_{-0.00}^{+0.73}$ & $108_{-26}^{+25}$ & 5082 & -6104 & -18859 \\
\hline NGC 4147 & 21.5 & $47_{-8}^{+9}$ & $-3_{-22}^{+12}$ & $136_{-5}^{+5}$ & $26.4_{-1.9}^{+0.6}$ & $0.4_{-0.0}^{+0.9}$ & $0.97_{-0.07}^{+0.00}$ & $93_{-12}^{+20}$ & 314 & -27 & -81117 \\
\hline NGC 4372 & 7.3 & $16_{-14}^{+11}$ & $133_{-9}^{+6}$ & $\begin{array}{r}150_{-7}^{+6} \\
\end{array}$ & $7.3_{-0.2}^{+0.3}$ & $3.0_{-0.3}^{+0.2}$ & $0.42_{-0.02}^{+0.04}$ & $28_{-2}^{+3}$ & 98 & 962 & -139570 \\
\hline Rup 106 & 18.5 & $-242_{-8}^{+5}$ & $91_{-15}^{+16}$ & $261_{-7}^{+12}$ & $37.9_{-5.1}^{+5.6}$ & $4.7_{-0.8}^{+0.9}$ & $0.78_{-0.03}^{+0.03}$ & $46_{-4}^{+\overline{6}}$ & 498 & 1640 & -64986 \\
\hline NGC 4590 & 10.3 & $\begin{array}{l}-169_{-12}^{+7} \\
\end{array}$ & $293_{-12}^{+6}$ & $339_{-6}^{+4}$ & $29.9_{-2.4}^{+1.4}$ & $8.9_{-0.4}^{+0.2}$ & $0.54_{-0.02}^{+0.02}$ & $41_{-3}^{+2}$ & 428 & 2453 & -70495 \\
\hline NGC 4833 & 7.2 & $105_{-15}^{+12}$ & $40_{-13}^{+12}$ & $120_{-13}^{+12}$ & $8.0_{-0.3}^{+0.4}$ & $0.7_{-0.3}^{+0.2}$ & $0.83_{-0.04}^{+0.07}$ & $36_{-8}^{+13}$ & 86 & 286 & -144428 \\
\hline NGC 5024 & 18.5 & $-95_{-6}^{+7}$ & $141_{-10}^{+8}$ & $184_{-10}^{+8}$ & $22.3_{-2.0}^{+2.1}$ & $8.9_{-1.4}^{+1.4}$ & $0.43_{-0.03}^{+0.03}$ & $74_{-2}^{+2}$ & 332 & 797 & -80241 \\
\hline NGC 5053 & 17.9 & $-89_{-3}^{+5}$ & $134_{-9}^{+5}$ & $164_{-8}^{+4}$ & $18.0_{-0.6}^{+1.1}$ & $10.4_{-1.1}^{+1.0}$ & $0.27_{-0.03}^{+0.04}$ & $76_{-1}^{+1}$ & 300 & 727 & -85221 \\
\hline NGC 5139 & 6.6 & $-70_{-4}^{+7}$ & $\begin{array}{l}-72_{-7}^{+6} \\
\end{array}$ & $128_{-9}^{+7}$ & $7.4_{-0.4}^{+0.1}$ & $1.1_{-0.1}^{+0.3}$ & $0.73_{-0.05}^{+0.02}$ & $137_{-5}^{-\frac{1}{7}}$ & 80 & -462 & -147850 \\
\hline NGC 5272 & 12.2 & $-38_{-5}^{+7}$ & $143_{-7}^{+10}$ & $200_{-6}^{+8}$ & $15.9_{-0.9}^{+0.7}$ & $5.2_{-0.3}^{+0.4}$ & $0.51_{-0.03}^{+0.01}$ & $57_{-2}^{+1}$ & 212 & 994 & -98226 \\
\hline NGC 5286 & 8.9 & $-220_{-4}^{+4}$ & $-44_{-15}^{+14}$ & $224_{-1}^{+3}$ & $13.7_{-0.7}^{+1.2}$ & $0.8_{-0.2}^{+0.4}$ & $0.89_{-0.04}^{+0.02}$ & $123_{-10}^{+10}$ & 154 & -375 & -113332 \\
\hline NGC 5634 & 21.1 & $-45_{-16}^{+12}$ & $39_{-16}^{+15}$ & $65_{-5}^{+11}$ & $21.6_{-1.1}^{+1.1}$ & $2.1_{-0.3}^{+0.9}$ & $0.82_{-0.06}^{+0.02}$ & $70_{-3}^{+\overline{7}}$ & 256 & 346 & -89143 \\
\hline NGC 5694 & 29.3 & $-185_{-13}^{+8}$ & $\begin{array}{r}-44_{-22}^{+18} \\
\end{array}$ & $252_{-10}^{+12}$ & $70.4_{-7.8}^{+10.4}$ & $2.5_{-1.0}^{+1.7}$ & $0.93_{-0.04}^{+0.03}$ & $136_{-17}^{+10}$ & 992 & -1019 & -45290 \\
\hline IC 4499 & 15.6 & $-245_{-4}^{+7}$ & $-75_{-14}^{+9}$ & $263_{-5}^{+4}$ & $30.0_{-2.7}^{+3.1}$ & $6.5_{-0.4}^{+0.8}$ & $0.65_{-0.05}^{+0.02}$ & $113_{-3}^{+4}$ & 406 & -1066 & -72235 \\
\hline NGC 5824 & 25.7 & $\begin{array}{l}-41_{-20}^{+17} \\
-{ }^{7}\end{array}$ & $105_{-24}^{+13}$ & $215_{-14}^{+11}$ & $37.4_{-4.3}^{+2.6}$ & $14.0_{-3.6}^{+1.7}$ & $0.45_{-0.05}^{+0.10}$ & $58_{-2}^{+4}$ & 598 & 2393 & -59779 \\
\hline Pal 5 & 18.4 & $-54_{-6}^{+5}$ & $160_{-43}^{+39}$ & $169_{-38}^{+37}$ & $18.9_{-0.6}^{+2.4}$ & $10.7_{-3.6}^{+4.3}$ & $0.28_{-0.13}^{+0.18}$ & $66_{-4}^{+4}$ & 308 & 1260 & -83194 \\
\hline NGC 5897 & 7.3 & $88_{-26}^{+13}$ & $97_{-23}^{+14}$ & $159_{-19}^{+8}$ & $8.7_{-0.4}^{+0.6}$ & $1.9_{-0.5}^{+0.4}$ & $0.64_{-0.05}^{+0.08}$ & $61_{-5}^{+5}$ & 106 & 362 & -131771 \\
\hline NGC 5904 & 6.3 & $-290+17$ & $126_{-14}^{+11}$ & $365_{-14}^{+13}$ & $23.3_{-3.3}^{+3.9}$ & $2.3_{-0.3}^{+0.5}$ & $0.82_{-0.03}^{+0.03}$ & $72_{-3}^{+3}$ & 286 & 402 & -85576 \\
\hline NGC 5927 & 4.7 & $-39_{-23}^{+20}$ & $233_{-11}^{+6}$ & $236_{-9}^{+6}$ & $5.2_{-0.2}^{+0.3}$ & $4.2_{-0.5}^{+0.4}$ & $0.11_{-0.05}^{+0.07}$ & $9_{-1}^{+1}$ & 82 & 1077 & -148662 \\
\hline NGC 5946 & 5.8 & $35_{-18}^{+6}$ & $25_{-15}^{+6}$ & $116_{-9}^{+9}$ & $5.9_{-0.2}^{+0.6}$ & $0.4_{-0.2}^{+0.2}$ & $0.89_{-0.05}^{+0.05}$ & $77_{-3}^{+8}$ & 66 & 141 & -158006 \\
\hline ESO $224-8$ & 12.6 & $-43_{-14}^{+60}$ & $259_{-22}^{+21}$ & $262_{-21}^{+19}$ & $17.0_{-2.7}^{+3.0}$ & $11.9_{-1.3}^{+0.9}$ & $0.18_{-0.07}^{+0.08}$ & $7_{-1}^{+1}$ & 292 & 3245 & -85205 \\
\hline NGC 5986 & 4.7 & $62_{-33}^{+21}$ & $23_{-15}^{+16}$ & $68_{-32}^{+\overline{2} 4}$ & $5.2_{-0.0}^{+0.6}$ & $0.2_{-0.1}^{+0.2}$ & $0.93_{-0.08}^{+0.05}$ & $66_{-14}^{+10}$ & 56 & 94 & -168505 \\
\hline FSR 1716 & 4.8 & $87_{-44}^{+47}$ & $228_{-15}^{+21}$ & $281_{-10}^{+24}$ & $7.0_{-0.7}^{+1.4}$ & $3.9_{-0.7}^{+0.7}$ & $0.28_{-0.07}^{+0.13}$ & $31_{-3}^{+2}$ & 106 & 1089 & -137191 \\
\hline Pal 14 & 71.4 & $117_{-18}^{+25}$ & $16_{-41}^{+16}$ & $177_{-17}^{+29}$ & $133.9_{-22.5}^{+31.3}$ & $2.2_{-0.4}^{+5.1}$ & $0.97_{-0.07}^{+0.00}$ & $50_{-0}^{+-63}$ & 2202 & 794 & -29458 \\
\hline BH 184 & 4.4 & $40_{-21}^{+15}$ & $121_{-11}^{+9}$ & $156_{-9}^{+8}$ & $4.7_{-0.4}^{+0.4}$ & $1.7_{-0.3}^{+0.2}$ & $0.47_{-0.04}^{+0.06}$ & $36_{-3}^{+3}$ & 58 & 531 & -168579 \\
\hline NGC 6093 & 3.7 & $33_{-17}^{+12}$ & $16_{-19}^{+9}$ & $71_{-7}^{+9}$ & $4.3_{-0.9}^{+0.0}$ & $0.2_{-0.1}^{+0.3}$ & $0.93_{-0}^{+0}$ & $83_{-5}^{+8}$ & 44 & 25 & -176933 \\
\hline NGC 6121 & 6.3 & $-52_{-2}^{+2}$ & $10_{-12}^{+18}$ & $54_{-2}^{+7}$ & $6.4_{-0.1}^{+0.2}$ & $0.2_{-0.1}^{+0.1}$ & $0.94_{-0.03}^{+0.04}$ & $21_{-31}^{+82}$ & 68 & 60 & -159021 \\
\hline NGC 6101 & 11.1 & $-12_{-12}^{+29}$ & $-314_{-2}^{+3}$ & $370_{-4}^{+6}$ & $44.2_{-4.8}^{+4.1}$ & $10.9_{-0.9}^{+0.4}$ & $0.61_{-0.02}^{+0.02}$ & $143_{-2}^{+2}$ & 658 & -3236 & -56722 \\
\hline NGC 6144 & 2.7 & $-69_{-93}^{+90}$ & $-196_{-15}^{+66}$ & $213_{-3}^{+7}$ & $3.2_{-0.0}^{+0.0}$ & $2.1_{-0.3}^{+0.2}$ & $0.21_{-0.01}^{+0.14}$ & $114_{-9}^{+8}$ & 40 & -239 & -172662 \\
\hline NGC 6139 & 3.5 & $-1_{-16}^{+16}$ & $76_{-8}^{+4}$ & $156_{-9}^{+9}$ & $3.6_{-0.1}^{+0.3}$ & $1.1_{-0.2}^{+0.2}$ & $0.54_{-0.03}^{+0.07}$ & $62_{-3}^{+4}$ & 52 & 248 & -176480 \\
\hline Terzan 3 & 2.5 & $\begin{array}{r}-61_{-51}^{+45} \\
\end{array}$ & $206_{-22}^{+7}$ & $236_{-8}^{+7}$ & $3.2_{-0.2}^{+0.4}$ & $\begin{array}{l}2.2_{-0.4}^{+0.1} \\
\end{array}$ & $0.18_{-0.03}^{+0.13}$ & $42_{-4}^{+6}$ & 44 & 440 & -175324 \\
\hline
\end{tabular}


Table 1: continued.

\begin{tabular}{|c|c|c|c|c|c|c|c|c|c|c|c|}
\hline Name & $\begin{array}{l}d_{G C} \\
{[\mathrm{kpc}]}\end{array}$ & $\begin{array}{r}\Pi \\
{[\mathrm{km} / \mathrm{s}]}\end{array}$ & $\begin{array}{r}\Theta \\
{[\mathrm{km} / \mathrm{s}]}\end{array}$ & $\begin{array}{r}V_{t o t} \\
{[\mathrm{~km} / \mathrm{s}]}\end{array}$ & $\begin{array}{r}\text { apo } \\
{[\mathrm{kpc}]}\end{array}$ & $\begin{array}{r}\text { peri } \\
{[\mathrm{kpc}]}\end{array}$ & ecc & $\begin{array}{r}\text { incl. } \theta \\
\text { [deg] }\end{array}$ & $\begin{array}{r}T_{r} \\
{[\mathrm{Myr}]}\end{array}$ & $\begin{array}{r}L_{Z} \\
{[\mathrm{kpc}} \\
\mathrm{km} / \mathrm{s}] \\
\end{array}$ & $\begin{array}{r}E \\
{\left[\mathrm{~km}^{2}\right.} \\
\left./ \mathrm{s}^{2}\right]\end{array}$ \\
\hline NGC 6171 & 3.5 & $-4_{-3}^{+7}$ & $78_{-8}^{+15}$ & $101_{-6}^{+13}$ & $3.8_{-0.3}^{+0.3}$ & $0.6_{-0.2}^{+0.3}$ & $0.72_{-0.11}^{+0.08}$ & $52_{-4}^{+7}$ & 44 & 191 & -178959 \\
\hline ESO 452-11 & 2.1 & $-24_{-15}^{+11}$ & $-13_{-11}^{+12}$ & $107_{-2}^{+10}$ & $2.9_{-0.1}^{+0.4}$ & $0.0_{-0.0}^{+0.1}$ & $0.97_{-0.03}^{+0.01}$ & $101_{-10}^{+7}$ & 26 & -16 & -202684 \\
\hline NGC 6205 & 8.6 & $20_{-4}^{+6}$ & $-26_{-7}^{+5}$ & $87_{-6}^{+5}$ & $8.6_{-0.2}^{+0.3}$ & $1.0_{-0.1}^{+0.2}$ & $0.79_{-0.03}^{+0.03}$ & $105_{-3}^{+4}$ & 100 & -187 & -134416 \\
\hline NGC 6229 & 29.9 & $30_{-12}^{+11}$ & $6_{-9}^{+8}$ & $59_{-13}^{+8}$ & $31.0_{-1.0}^{+1.1}$ & $0.5_{-0.1}^{+0.6}$ & $0.97_{-0.04}^{+0.01}$ & $77_{-21}^{+26}$ & 376 & 135 & -74434 \\
\hline NGC 6218 & 4.8 & $-9_{-9}^{+3}$ & $135_{-10}^{+6}$ & $158_{-7}^{+6}$ & $5.0_{-0.1}^{+0.4}$ & $2.3_{-0.2}^{+0.3}$ & $0.37_{-0.02}^{+0.05}$ & $37_{-2}^{+2}$ & 64 & 581 & -158135 \\
\hline FSR 1735 & 4.3 & $-102_{-7}^{+17}$ & $-5_{-21}^{+15}$ & $225_{-12}^{+16}$ & $5.3_{-0.3}^{+0.6}$ & $1.0_{-0.1}^{+0.5}$ & $0.69_{-0.10}^{+0.03}$ & $92_{-4}^{+6}$ & 68 & -22 & -157538 \\
\hline NGC 6235 & 4.0 & $159_{-5}^{+8}$ & $197_{-40}^{+28}$ & $256_{-30}^{+24}$ & $6.2_{-1.1}^{+1.2}$ & $2.7_{-0.5}^{+0.4}$ & $0.39_{-0.03}^{+0.05}$ & $53_{-7}^{+11}$ & 84 & 570 & -145439 \\
\hline NGC 6254 & 4.8 & $-87_{-7}^{+4}$ & $134_{-16}^{+13}$ & $167_{-10}^{+9}$ & $5.2_{-0.3}^{+0.2}$ & $2.1_{-0.4}^{+0.3}$ & $0.42_{-0.06}^{+0.06}$ & $36_{-1}^{+4}$ & 78 & 606 & -157472 \\
\hline NGC 6256 & 2.9 & $-170_{-0}^{+18}$ & $28_{-18}^{+39}$ & $198_{-11}^{+4}$ & $4.4_{-0.8}^{+0.9}$ & $0.1_{-0.1}^{+0.3}$ & $0.94_{-0.15}^{+0.04}$ & $78_{-14}^{+\overline{7}^{1}}$ & 46 & 79 & -181873 \\
\hline Pal 15 & 38.2 & $154_{-13}^{+9}$ & $\begin{array}{l}-5_{-17}^{+26} \\
-{ }_{-17}\end{array}$ & $162_{-11}^{+9}$ & $\begin{array}{r}54.6_{-4.4}^{+2.8} \\
\end{array}$ & $1.2_{-0.3}^{+3.0}$ & $0.96_{-0.11}^{+0.01}$ & $98_{-35}^{+18}$ & 726 & -165 & -53233 \\
\hline NGC 6266 & 2.0 & $42_{-15}^{+17}$ & $122_{-18}^{+10}$ & $146_{-11}^{+6}$ & $2.5_{-0.4}^{+0.5}$ & $0.6_{-0.1}^{+0.3}$ & $0.62_{-0.10}^{+0.04}$ & $32_{-4}^{+4}$ & 32 & 215 & -205537 \\
\hline NGC 6273 & 1.6 & $-98_{-143}^{+67}$ & $-240_{-34}^{+218}$ & $315_{-4}^{+8}$ & $3.8_{-0.3}^{+0.6^{+0}}$ & $1.0_{-0.1}^{+0.13}$ & $0.59_{-0.08}^{+0.11}$ & $109_{-18}^{+10}$ & 48 & -144 & -172941 \\
\hline NGC 6284 & 7.3 & $14_{-2}^{+2}$ & $-2_{-19}^{+7}$ & $113_{-6}^{+12}$ & $\begin{array}{r}7.5_{-0.9}^{+0.9} \\
\end{array}$ & $\begin{array}{l}0.7_{-0.2}^{+0.1} \\
+0.3\end{array}$ & $0.83_{-0.06}^{+0.08}$ & $91_{-4}^{+10}$ & 90 & -16 & -142332 \\
\hline NGC 6287 & 2.0 & $-301_{-107}^{+26 \overline{4}}$ & $-64_{-80}^{+40}$ & $318_{-4}^{+5}$ & $5.3_{-0.5}^{+0.6}$ & $0.8_{-0}^{+0}$ & $0.75_{-0.02}^{+0.04}$ & $95_{-3}^{+3}$ & 66 & -60 & -159009 \\
\hline NGC 6293 & 1.8 & $-152_{-33}^{+52}$ & $-80_{-36}^{+115}$ & $233_{-10}^{+7^{2}}$ & $3.6_{-1.0}^{+0.4}$ & $0.2_{-0}^{+0}$ & $0.91_{-0.17}^{+0.08}$ & $131_{-39}^{+7^{\circ}}$ & 38 & -93 & -191358 \\
\hline NGC 6304 & 2.5 & $79_{-10}^{+5}$ & $191_{-6}^{+7}$ & $219_{-6}^{+5}$ & $3.3_{-0.6}^{+0.4}$ & $1.8_{-0}^{+0}$ & $0.29_{-0.02}^{+0.04}$ & $20_{-2}^{+2}$ & 52 & 474 & -183132 \\
\hline NGC 6316 & 2.4 & $103_{-7}^{+9}$ & $51_{-33}^{+26}$ & $143_{-11}^{+16}$ & $3.0_{-0.6}^{+0.5}$ & $0.4_{-0}^{+0}$ & $0.77_{-0.03}^{+0.18}$ & $40_{-16}^{+31}$ & 36 & 106 & -197316 \\
\hline NGC 6341 & 9.8 & $53_{-4}^{+6}$ & $13_{-5}^{+9}$ & $109_{-13}^{+12}$ & $10.7_{-0.3}^{+0.3}$ & $0.3_{-0}^{+0}$ & $0.94_{-0.04}^{+0.02}$ & $\begin{array}{l}79_{-9}^{+4} \\
-4\end{array}$ & 124 & 108 & -125312 \\
\hline NGC 6325 & 1.3 & $\begin{array}{l}-81_{-48}^{+29} \\
\end{array}$ & $-181_{-63}^{+187}$ & $214_{-19}^{+19}$ & $1.3_{-0.1}^{+0.4}$ & $1.1_{-0.3}^{+0.2}$ & $0.12_{-0.00}^{+0.16}$ & $114_{-25}^{+13}$ & 18 & -107 & -212097 \\
\hline NGC 6333 & 1.8 & $-89_{-118}^{+122}$ & $346_{-48}^{+6}$ & $364_{-3}^{+5}$ & $6.4_{-0.2}^{+0.8}$ & $1.0_{-0.1}^{+0.2}$ & $0.74_{-0.03}^{+0.02}$ & $59_{-6}^{+2}$ & 74 & 327 & -151409 \\
\hline NGC 6342 & 1.6 & $\begin{array}{l}-25_{-89}^{+70} \\
-10\end{array}$ & $164_{-66}^{+13}$ & $168_{-4}^{+6}$ & $1.7_{-0.3}^{+0.7}$ & $0.9_{-0.4}^{+0.2}$ & $0.31_{-0.12}^{+0.29}$ & $64_{-3}^{+6}$ & 24 & 117 & -207010 \\
\hline NGC 6356 & 7.2 & $47_{-9}^{+5}$ & $107_{-12}^{+25}$ & $160_{-7}^{+17}$ & $7.9_{-1.1}^{+1.3}$ & $2.5_{-0.5}^{+1.0}$ & $0.52_{-0.10}^{+0.06}$ & $43_{-4}^{+3}$ & 104 & 713 & -136303 \\
\hline NGC 6355 & 1.2 & $-207_{-47}^{+70}$ & $-110_{-54}^{+108}$ & $275_{-10}^{+8}$ & $2.2_{-0.5}^{+1.1}$ & $0.6_{-0.2}^{+0.2}$ & $0.56_{-0.10}^{+0.17}$ & $106_{-10}^{+8}$ & 28 & -95 & -199192 \\
\hline NGC 6352 & 3.6 & $42_{-13}^{+18}$ & $226_{-13}^{+5}$ & $230_{-10}^{+4}$ & $4.1_{-0.3}^{+0.6}$ & $3.2_{-0.3}^{+0.2}$ & $0.13_{-0.03}^{+0.05}$ & $12_{-1}^{+1}$ & 68 & 794 & -163864 \\
\hline IC 1257 & 17.6 & $-45_{-12}^{+7}$ & $-50_{-18}^{+12}$ & $70_{-0}^{+12}$ & $18.1_{-0.8}^{+1.1}$ & $1.8_{-0.4}^{+0.8}$ & $0.82_{-0.08}^{+0.04}$ & $158_{-11}^{+4}$ & 208 & -817 & -98556 \\
\hline Terzan 2 & 1.0 & $-120_{-23}^{+62}$ & $-47_{-48}^{+13}$ & $136_{-2}^{+3}$ & $1.2_{-0.4}^{+0.9}$ & $0.1_{-0.8}^{+0 .}$ & $0.86_{-0.19}^{+0.05}$ & $161_{-19}^{+3}$ & 14 & -44 & -242360 \\
\hline NGC 6366 & 5.3 & $94_{-3}^{+3}$ & $134_{-6}^{+2}$ & $175_{-5}^{+2}$ & $5.8_{-0.2}^{+0.2}$ & $2.2_{-0}^{+0}$ & $0.45_{-0.00}^{+0.03}$ & $32_{-1}^{+2}$ & 74 & 699 & -153378 \\
\hline Terzan 4 & 1.2 & $15_{-39}^{+\overline{8}^{3}}$ & $75_{-13}^{+18}$ & $124_{-8}^{+14}$ & $1.3_{-0.4}^{+0.2}$ & $0.2_{-0.1}^{+0.1}$ & $0.68_{-0.03}^{+0.03}$ & $52_{-6}^{+\frac{1}{+7}}$ & 14 & 92 & -234494 \\
\hline BH 229 & 0.5 & $7_{-39}^{+46}$ & $-55_{-0}^{+48}$ & $292_{-22}^{+14}$ & $0.8_{-0.1}^{+1.4}$ & $0.3_{-0}^{+0}:$ & $0.49_{-0.18}^{+0.32}$ & $100_{-9}^{+0}$ & 10 & -21 & -249808 \\
\hline FSR 1758 & 3.7 & $60_{-60}^{+30}$ & $\begin{array}{r}-8 \\
-347_{-6}^{+8}\end{array}$ & $405_{-8}^{+8}$ & $14.3_{-2.1}^{+2.1}$ & $3.7_{-0}^{+0}$ & $0.59_{-0.02}^{+0.03}$ & $148_{-2}^{+2}$ & 178 & -1275 & -106508 \\
\hline NGC 6362 & 5.2 & $\begin{array}{l}17_{-28}^{+14} \\
\end{array}$ & $124_{-6}^{+11}$ & $160_{-4}^{+11}$ & $5.3_{-0.4}^{+0.1}$ & $2.5_{-0}^{+0}$ & $0.37_{-0.04}^{+0.04}$ & $45_{-4}^{+2}$ & 70 & 583 & -153468 \\
\hline Liller 1 & 0.8 & $107_{-57}^{+25}$ & $-56_{-43}^{+61}$ & $123_{-31}^{+28}$ & $0.8_{-0.1}^{+0.2}$ & $0.1_{-0}^{+0}$ & $0.81_{-0.16}^{+0.13}$ & $155_{-74}^{+16}$ & 8 & -42 & -261395 \\
\hline NGC6380 & 3.1 & $-62_{-14}^{+5}$ & $-35_{-9}^{+16}$ & $72_{-7}^{+14}$ & $3.4_{-0.6}^{+0.1}$ & $0.2_{-0}^{+0 .}$ & $0.89_{-0.07}^{+0.05}$ & $168_{-26}^{+11}$ & 40 & -105 & -194646 \\
\hline Terzan 1 & 1.6 & $-73_{-5}^{+3}$ & $63_{-20}^{+10}$ & $96_{-11}^{+8}$ & $1.8_{-0.6}^{+0.5}$ & $0.2_{-0.1}^{+0.1}$ & $0.79_{-0.04}^{+0.06}$ & $11_{-6}^{+10}$ & 22 & 102 & -224803 \\
\hline Pismis 26 & 1.4 & $-112_{-58}^{+111}$ & $204_{-55}^{+32}$ & $307_{-8}^{+9}$ & $\begin{array}{l}3.2_{-0.1}^{+0.6} \\
0.8\end{array}$ & $0.9_{-0.4}^{-0.1}$ & $0.56_{-0.20}^{+0.04}$ & $\begin{array}{r}-6 \\
41_{-2}^{+9}\end{array}$ & 44 & 271 & -186876 \\
\hline NGC 6388 & 3.0 & $\begin{array}{l}-66_{-19}^{+24} \\
\end{array}$ & $-94_{-12}^{+18}$ & $116_{-7}^{+6}$ & $3.5_{-0.2}^{+0.1}$ & $0.7_{-0.2}^{+0.4}$ & $0.69_{-0.13}^{+0.07}$ & $148_{-9}^{+8}$ & 46 & -257 & -190150 \\
\hline NGC 6402 & 4.0 & $-20_{-21}^{+23}$ & $48_{-7}^{+10}$ & $57_{-7}^{+16}$ & $4.8_{-}^{+}$ & $0.3_{-0}^{+0}$ & $0.88_{-0}^{+0}$ & $47_{-10}^{+\overline{6}^{3}}$ & 52 & 158 & -176457 \\
\hline NGC 6401 & 2.5 & $-30_{-18}^{+27}$ & $-254_{-4}^{+4}$ & $302_{-4}^{+8}$ & $4.5_{-0}^{+0}$ & $2.4_{-0}^{+0}$ & $0.31_{-0}^{+0}$ & $143_{-3}^{+2}$ & 62 & -595 & -161761 \\
\hline NGC 6397 & 6.3 & $35_{-6}^{+7}$ & $127_{-6}^{+11}$ & $179_{-4}^{+9}$ & $6.5_{-0.2}^{+0.1}$ & $2.8_{-\varepsilon}^{+c}$ & $0.40_{-0}^{+0}$ & $43_{-4}^{+2}$ & 86 & 796 & -144538 \\
\hline Pal 6 & 2.5 & $-191_{-3}^{+2}$ & $21_{-14}^{+16}$ & $246_{-8}^{+6}$ & $4.5_{-0.8}^{+0.4}$ & $0.1_{-0}^{+0}$ & $0.96_{-0}^{+0}$ & $83_{-6}^{+4}$ & 44 & 52 & -179351 \\
\hline NGC 6426 & 14.3 & $-112_{-16}^{+27}$ & $93_{-20}^{+7}$ & $148_{-25}^{+11}$ & $16.6_{-0.7}^{+1.0}$ & $3.2_{-0}^{+0}$ & $0.67_{-0}^{+0}$ & $27_{-4}^{+4}$ & 202 & 1216 & -100666 \\
\hline Djorg 1 & 1.2 & $\begin{array}{l}-252_{-102}^{+270} \\
\end{array}$ & $315_{-51}^{+49}$ & $404_{-9}^{+12}$ & $5.9_{-1.6}^{+1.6}$ & $0.8_{-0}^{+0 .}$ & $0.76_{-0.06}^{+0.05}$ & $21_{-7}^{+13}$ & 66 & 351 & -161437 \\
\hline Terzan 5 & 1.5 & $84_{-6}^{+11}$ & $70_{-23}^{+13}$ & $114_{-7}^{+9}$ & $1.7_{-0.6}^{+0.5}$ & $0.2_{-0}^{+0}:$ & $0.78_{-0.05}^{+0.04}$ & $33_{-7}^{+18}$ & 20 & 104 & -226313 \\
\hline NGC 6440 & 1.3 & $91_{-36}^{+18}$ & $-42_{-34}^{+54}$ & $107_{-8}^{+10}$ & $1.4_{-0.4}^{+0.4}$ & $0.2_{-0}^{+0}$ & $0.78_{-0.15}^{+0.15}$ & $116_{-34}^{+21}$ & 14 & -49 & -231796 \\
\hline NGC 6441 & 3.6 & $16_{-8}^{+15}$ & $\begin{array}{l}66_{-19}^{+18} \\
\end{array}$ & $71_{-16}^{+20}$ & $3.6_{-0.6}^{+0.7}$ & $0.8_{-0.2}^{+0.2}$ & $0.66_{-0.07}^{+0.09}$ & $21_{-6}^{+11}$ & 42 & 228 & -186312 \\
\hline Terzan 6 & 1.5 & $-138_{-3}^{+7}$ & $\begin{array}{l}-51_{-32}^{+21} \\
-21\end{array}$ & $153_{-3}^{+9}$ & $2.0_{-0}^{+0}$ & $0.1_{-0}^{+0}$ & $0.86_{-0.11}^{+0.05}$ & $157_{-26}^{+17}$ & 22 & -77 & -220185 \\
\hline NGC 6453 & 3.4 & $\begin{array}{l}-105_{-6}^{+9} \\
-9\end{array}$ & $38_{-15}^{+30}$ & $194_{-5}^{+19}$ & $3.9_{-0}^{+0}$ & $0.9_{-0}^{+0}$ & $0.61_{-0.14}^{+0.04}$ & $78_{-8}^{+5}$ & 54 & 129 & -172906 \\
\hline NGC 6496 & 4.0 & $\begin{array}{l}-37_{-63}^{+76} \\
-7\end{array}$ & $320_{-41}^{+24}$ & $328_{-28}^{+26}$ & $9.1_{-1.6}^{+2.0}$ & $3.7_{-0}^{+0}:$ & $0.42_{-0.07}^{+0.10}$ & $32_{-3}^{+5}$ & 120 & 1111 & -126509 \\
\hline Terzan 9 & 1.3 & $-50_{-7}^{+8}$ & $22_{-14}^{+18}$ & $76_{-2}^{+7}$ & $1.4_{-0.4}^{+0.6}$ & $0.1_{-0.0}^{+0.1}$ & $0.92_{-0.08}^{+0.03}$ & $70_{-13}^{+12}$ & 16 & 29 & -236057 \\
\hline Djorg 2 & 2.0 & $161_{-6}^{+9}$ & $155_{-11}^{+6}$ & $228_{-4}^{+3}$ & $3.2_{-0.7}^{+0.6}$ & $0.9_{-0.3}^{+0.0}$ & $0.57_{-0.04}^{+0.06}$ & $11_{-1}^{+1}$ & 42 & 316 & -192751 \\
\hline NGC 6517 & 4.0 & $55_{-17}^{+8}$ & $33_{-12}^{+10}$ & $73_{-11}^{+\overline{5}^{4}}$ & $4.6_{-0.4}^{+0.4}$ & $0.2_{-0.1}^{+0.1}$ & $0.91_{-0.04}^{+0.03}$ & $58_{-10}^{+10}$ & 50 & 127 & -179281 \\
\hline Terzan 10 & 2.2 & $231_{-52}^{+20}$ & $87_{-22}^{+64}$ & $343_{-13}^{+20}$ & $5.9_{-1.8}^{+1.4}$ & $0.7_{-0.1}^{+0.3}$ & $0.79_{-0.04}^{+0.04}$ & $72_{-10}^{+4}$ & 76 & 193 & -155422 \\
\hline
\end{tabular}


Table 1: continued.

\begin{tabular}{|c|c|c|c|c|c|c|c|c|c|c|c|}
\hline Name & $\begin{array}{c}d_{G C} \\
{[\mathrm{kpc}]}\end{array}$ & $\begin{array}{r}\Pi \\
{[\mathrm{km} / \mathrm{s}]}\end{array}$ & $\begin{array}{r}\Theta \\
{[\mathrm{km} / \mathrm{s}]}\end{array}$ & $\begin{array}{r}V_{t o t} \\
{[\mathrm{~km} / \mathrm{s}]}\end{array}$ & $\begin{array}{r}\text { apo } \\
{[\mathrm{kpc}]}\end{array}$ & $\begin{array}{r}\text { peri } \\
{[\mathrm{kpc}]}\end{array}$ & ecc & $\begin{array}{r}\text { incl. } \theta \\
\text { [deg] }\end{array}$ & $\begin{array}{r}T_{r} \\
{[\mathrm{Myr}]}\end{array}$ & $\begin{array}{r}L_{Z} \\
{[\mathrm{kpc}} \\
\mathrm{km} / \mathrm{s}]\end{array}$ & $\begin{array}{r}E \\
{\left[\mathrm{~km}^{2}\right.} \\
\left./ \mathrm{s}^{2}\right]\end{array}$ \\
\hline NGC 6522 & 0.8 & $34_{-15}^{+25}$ & $92_{-57}^{+23}$ & $213_{-9}^{+17}$ & $1.2_{-0.3}^{+0.5}$ & $0.2_{-0.2}^{+0.3}$ & $0.67_{-0.15}^{+0.14}$ & $63_{-6}^{+16}$ & 16 & 58 & -238519 \\
\hline NGC 6535 & 4.0 & $93_{-10}^{+9}$ & $-83_{-6}^{+13}$ & $133_{-6}^{+2}$ & $4.6_{-0.3}^{+0.4}$ & $1.0_{-0.2}^{+0.1}$ & $0.64_{-0.03}^{+0.06}$ & $160_{-3}^{+1}$ & 56 & -320 & -173024 \\
\hline NGC 6528 & 0.7 & $-197_{-67}^{+300}$ & $113_{-67}^{+31}$ & $229^{+6}$ & $1.0^{+0.9}$ & $0.3^{+0.0}$ & $0.60^{+0.33}$ & $70_{-5}^{+3}$ & 14 & 51 & -241883 \\
\hline NGC 6539 & 3.1 & $1_{-9}^{+28}$ & $118_{-3}^{+5}$ & $208_{-9}^{+14}$ & $3.4_{-0.2}^{+0.3}$ & $1.9^{+0.2}$ & $0.30_{-0.03}^{+0.08}$ & $56_{-2}^{+1}$ & 56 & 347 & -174387 \\
\hline NGC 6540 & 3.0 & $\begin{array}{r}13_{-2}^{+3} \\
-3\end{array}$ & $148_{-7}^{+9}$ & $159_{-5}^{+8}$ & $3.1_{-0.4}^{+0.2}$ & $1.6_{-0.2}^{+0.3}$ & $0.32_{-0.04}^{+0.03}$ & $22_{-1}^{+2}$ & 48 & 448 & -187517 \\
\hline NGC 6544 & 5.3 & $6_{-2}^{+2}$ & $6_{-10}^{+14}$ & $91_{-7}^{+8}$ & $5.6_{-0.1}^{+0.2}$ & $0.1_{-0.0}^{+0.2}$ & $0.98_{-0.06}^{+0.00}$ & $86_{-9}^{+\frac{1}{+7}}$ & 52 & 31 & -166630 \\
\hline NGC 6541 & 2.3 & $123_{-62}^{+33}$ & $192_{-20}^{+25}$ & $254_{-6}^{+11}$ & $3.8_{-0.6}^{+0.6}$ & $1.3_{-0.1}^{+0.2}$ & $0.50_{-0.10}^{+0.08}$ & $40_{-4}^{+6}$ & 50 & 334 & -174968 \\
\hline ESO 280-06 & 13.8 & $31_{-10}^{+5}$ & $16_{-25}^{+10}$ & $91_{-11}^{+8}$ & $14.2_{-1.3}^{+1.1}$ & $0.9_{-0.4}^{+0.5}$ & $0.88_{-0.05}^{+0.05}$ & $77_{-7}^{+20}$ & 164 & 210 & -109791 \\
\hline NGC 6553 & 2.4 & $45_{-12}^{+15}$ & $245_{-4}^{+2}$ & $250_{-3}^{+2}$ & $3.3_{-0.4}^{+0.3}$ & $2.3_{-0.5}^{+0.4}$ & $0.19_{-0.04}^{+0.04}$ & $7_{-1}^{+2}$ & 52 & 588 & -179839 \\
\hline NGC 6558 & 1.2 & $187_{-94}^{+60}$ & $93_{-60}^{+36}$ & $209_{-4}^{+4}$ & $1.7_{-0.6}^{+0.4}$ & $0.3_{-0.0}^{+0.2}$ & $0.72_{-0.29}^{+0.08}$ & $63_{-8}^{+15}$ & 20 & 87 & -217130 \\
\hline Pal 7 & 3.9 & $-74_{-9}^{+12}$ & $270_{-4}^{+7}$ & $281_{-4}^{+7}$ & $6.0_{-0.4}^{+0.6}$ & $3.5_{-0.1}^{+0.0}$ & $0.26_{-0.02}^{+0.02}$ & $11_{-1}^{+1}$ & 86 & 1042 & -147032 \\
\hline Terzan 12 & 3.6 & $-94_{-4}^{+5}$ & $172_{-9}^{+8}$ & $219_{-6}^{+6}$ & $4.4_{-0.5}^{+0.4}$ & $2.2_{-0.3}^{+0 . \frac{1}{2}}$ & $0.33_{-0.03}^{+0.04}$ & $28_{-2}^{+\frac{1}{2}}$ & 62 & 625 & -167749 \\
\hline NGC 6569 & 2.8 & $-40_{-4}^{+2}$ & $174_{-26}^{+18}$ & $180_{-25}^{+18}$ & $3.0_{-0.7}^{+0.6}$ & $1.9_{-0.7}^{+0.3}$ & $0.23_{-0.05}^{+0.17}$ & $26_{-5}^{+12}$ & 56 & 440 & -182361 \\
\hline ESO $456-78$ & 2.0 & $71_{-10}^{+10}$ & $199_{-4}^{+5}$ & $252_{-6}^{+7}$ & $2.9_{-0.2}^{+0.6}$ & $1.4_{-0.3}^{+0.5}$ & $0.34_{-0.07}^{+0.06}$ & $34_{-2}^{+2}$ & 52 & 373 & -186632 \\
\hline NGC 6584 & 6.8 & $197_{-30}^{+7}$ & $98_{-34}^{+22}$ & $324_{-22}^{+11}$ & $18.0_{-2.8}^{+2.1}$ & $1.7_{-0.6}^{+0.7}$ & $0.83_{-0.06}^{+0.05}$ & $52_{-3}^{+12}$ & 212 & 556 & -98089 \\
\hline NGC 6624 & 1.2 & $-29_{-24}^{+48}$ & $60_{-18}^{+14}$ & $136_{-4}^{+7}$ & $1.5_{-0.0}^{+0.8}$ & $0.2_{-0.2}^{+0.0}$ & $0.78_{-0.05}^{+0.00}$ & $\begin{array}{r}-3 \\
73_{-6}^{+3}\end{array}$ & 20 & 37 & -226410 \\
\hline NGC 6626 & 3.0 & $-28_{-4}^{+2}$ & $57_{-12}^{+9^{\circ}}$ & $113_{-5}^{+8}$ & $3.1_{-0.1}^{+0.5}$ & $0.5_{-0.2}^{+0.1}$ & $0.75_{-0.04}^{+0.10}$ & $60_{-4}^{+6}$ & 42 & 169 & -193067 \\
\hline NGC 6638 & 2.0 & $68_{-12}^{+16}$ & $14_{-21}^{+13}$ & $74_{-7}^{+14}$ & $2.4_{-0.1}^{+0.5}$ & $0.1_{-0.0}^{+0.0}$ & $0.95_{-0.03}^{+0.03}$ & $80_{-9}^{+16}$ & 20 & 22 & -212068 \\
\hline NGC 6637 & 1.6 & $35_{-111}^{+27}$ & $91_{-81}^{+61}$ & $\begin{array}{r}129_{-8}^{+8} \\
-8\end{array}$ & $2.2_{-0.4}^{+0.1}$ & $0.2_{-0.1}^{+0.0}$ & $0.87_{-0.01}^{+0.11}$ & $77_{-5}^{+17}$ & 22 & 40 & -212500 \\
\hline NGC 6642 & 1.7 & $112_{-22}^{+6}$ & $25_{-51}^{+32}$ & $125_{-11}^{+8}$ & $2.2_{-0.1}^{+0.3}$ & $0.1_{-0.0}^{+0 . \frac{1}{2}}$ & $0.94_{-0.11}^{+0.02}$ & $46_{-17}^{+78}$ & 24 & 36 & -215457 \\
\hline NGC 6652 & 2.5 & $-54_{-4}^{+6}$ & $28_{-18}^{+7}$ & $186_{-16}^{+11}$ & $4.2_{-0.3}^{+0.2}$ & $0.1_{-0.1}^{+0.1}$ & $0.96_{-0.05}^{+0.03}$ & $76_{-5}^{+9}$ & 38 & 42 & -183595 \\
\hline NGC 6656 & 5.2 & $176_{-2}^{+3}$ & $201_{-2}^{+2}$ & $303_{-5}^{+6}$ & $9.8_{-0.5}^{+0.7}$ & $3.1_{-0.1}^{+0.2}$ & $0.53_{-0.01}^{+0.01}$ & $33_{-2}^{+2}$ & 126 & 1044 & -125471 \\
\hline Pal 8 & 5.3 & $-21_{-25}^{+13}$ & $117_{-15}^{+23}$ & $122_{-15}^{+27}$ & $5.6_{-0.6}^{+0.3}$ & $1.8_{-0.4}^{+0.6}$ & $0.51_{-0.11}^{+0.06}$ & $23_{-2}^{+3}$ & 72 & 593 & -160020 \\
\hline NGC 6681 & 2.0 & $221_{-166}^{+34}$ & $55_{-44}^{+135}$ & $287_{-3}^{+9}$ & $4.5_{-0.4}^{+0.3}$ & $0.7_{-0.1}^{+0.5}$ & $0.74_{-0.15}^{+0.10}$ & $84_{-9}^{+8}$ & 52 & 36 & -167768 \\
\hline NGC 6712 & 3.6 & $146_{-7}^{+6}$ & $26_{-17}^{+9}$ & $208_{-10}^{+7}$ & $\begin{array}{l}5.5_{-0.3}^{+0.4} \\
\end{array}$ & $0.2_{-0.1}^{+0.1}$ & $0.93_{-0.02}^{+0.04}$ & $78_{-4}^{+7}$ & 56 & 93 & -168544 \\
\hline NGC 6715 & 18.6 & $232_{-8}^{+6}$ & $49_{-22}^{+25}$ & $317_{-11}^{+21}$ & $56.4_{-10.1}^{+24.2}$ & $14.8_{-1.0}^{+1.8}$ & $0.58_{-0.03}^{+0.07}$ & $81_{-4}^{+4}$ & 906 & 850 & -48159 \\
\hline NGC 6717 & 2.5 & $-10_{-13}^{+31}$ & $116_{-9}^{+8}$ & $119_{-7}^{+9}$ & $2.8_{-0.4}^{+0.4}$ & $0.7_{-0.1}^{+0.2}$ & $0.59_{-0.09}^{+0.06}$ & $32_{-4}^{+7}$ & 36 & 251 & -195762 \\
\hline NGC 6723 & 2.6 & $100_{-192}^{+6}$ & $-178_{-3}^{+353}$ & $208_{-5}^{+5}$ & $3.1_{-0.0}^{+0.4}$ & $1.8_{-0.0}^{+0.2}$ & $0.26_{-0.03}^{+0.05}$ & $90_{-12}^{+8}$ & 40 & -2 & -175356 \\
\hline NGC 6749 & 5.0 & $\begin{array}{l}+23_{-10}^{+17} \\
\end{array}$ & $110_{-9}^{+10}$ & $112_{-8}^{+10}$ & $5.1_{-0.2}^{+0.4}$ & $1.6_{-0.1}^{+0.2}$ & $0.53_{-0.05}^{+0.05}$ & $3_{-0}^{+1}$ & 62 & 556 & -167068 \\
\hline NGC 6752 & 5.5 & $\begin{array}{l}+73_{-2}^{+7} \\
-\end{array}$ & $179_{-6}^{+3}$ & $190_{-6}^{+3}$ & $5.7_{-0.3}^{+0.3}$ & $3.6_{-0.2}^{+0 . \frac{1}{2}}$ & $0.23_{-0.02}^{+0.02}$ & $24_{-0}^{+1}$ & 82 & 931 & -147164 \\
\hline NGC 6760 & 5.0 & $92_{-9}^{+10}$ & $147_{-8}^{+7}$ & $174_{-7}^{+7}$ & $5.6_{-0.3}^{+0.3}$ & $2.2_{-0.2}^{+0.2}$ & $0.44_{-0.03}^{+0.04}$ & $6_{-0}^{+1}$ & 72 & 724 & -158732 \\
\hline NGC 6779 & 9.3 & $\begin{array}{l}155_{-2}^{+3} \\
\end{array}$ & $-15_{-9}^{+6}$ & $185_{-4}^{+6}$ & $12.4_{-0.6}^{+0.4}$ & $0.3_{-0.1}^{+0.2}$ & $0.96_{-0.03}^{+0.01}$ & $101_{-5}^{+7}$ & 134 & -135 & -119696 \\
\hline Terzan 7 & 15.3 & $260_{-6}^{+6}$ & $25_{-11}^{+17}$ & $320_{-11}^{+11}$ & $42.9_{-8.3}^{+7.7}$ & $12.8_{-1.1}^{+0.6}$ & $0.54_{-0.05}^{+0.05}$ & $86_{-3}^{+2}$ & 668 & 335 & -56545 \\
\hline Pal 10 & 6.6 & $-56_{-5}^{+15}$ & $186_{-13}^{+8}$ & $195_{-14}^{+7}$ & $7.0_{-0.3}^{+0.3}$ & $4.0_{-0.4}^{+0.4}$ & $0.27_{-0.03}^{+0.04}$ & $8_{-1}^{+1}$ & 100 & 1234 & -138495 \\
\hline Arp 2 & 21.2 & $243_{-8}^{+7}$ & $68_{-11}^{+20}$ & $311_{-15}^{+10}$ & $65.1_{-13.5}^{+21.7}$ & $17.8_{-1.6}^{+1.7}$ & $0.57_{-0.04}^{+0.06}$ & $78_{-4}^{+2}$ & 1094 & 1256 & -43628 \\
\hline NGC 6809 & 4.1 & $-199_{-7}^{+5}$ & $76_{-19}^{+15}$ & $220_{-3}^{+5}$ & $5.7_{-0.5}^{+0.4}$ & $1.2_{-0.3}^{+0.3}$ & $0.66_{-0.07}^{+0.06}$ & $67_{-3}^{+6}$ & 76 & 266 & -154417 \\
\hline Terzan 8 & 19.1 & $269_{-5}^{+8}$ & $37_{-23}^{+16}$ & $315_{-7}^{+12}$ & $58.5_{-6.8}^{+13.5}$ & $16.0_{-0.8}^{+0.8}$ & $0.57_{-0.03}^{+0.06}$ & $84_{-3}^{+4}$ & 958 & 584 & -46785 \\
\hline Pal 11 & 8.1 & $-16_{-20}^{+28}$ & $139_{-10}^{+12}$ & $140_{-9}^{+14}$ & $8.2_{-0.4}^{+0.7}$ & $3.5_{-0.4}^{+0.6}$ & $0.40_{-0.04}^{+0.05}$ & $27_{-2}^{+3}$ & 108 & 1013 & -132057 \\
\hline NGC 6838 & 7.0 & $39_{-11}^{+5}$ & $204_{-4}^{+5}$ & $212_{-4}^{+4}$ & $7.3_{-0.2}^{+0.2}$ & $5.0_{-0.2}^{+0.3}$ & $0.18_{-0.03}^{+0.02}$ & $12_{-1}^{+1}$ & 112 & 1423 & -132307 \\
\hline NGC 6864 & 14.6 & $-99_{-6}^{+14}$ & $18_{-25}^{+10}$ & $111_{-14}^{+7}$ & $16.4_{-0.4}^{+1.3}$ & $0.5_{-0.5}^{+0.6}$ & $0.94_{-0.05}^{+0.06}$ & $61_{-9}^{+41}$ & 186 & 209 & -103615 \\
\hline NGC 6934 & 12.7 & $-289_{-18}^{+20}$ & $103_{-30}^{+20}$ & $330_{-17}^{+15}$ & $40.9_{-5.8}^{+7.8}$ & $2.5_{-0.8}^{+0.6}$ & $0.88_{-0.03}^{+0.04}$ & $23_{-2}^{+5}$ & 520 & 1204 & -63341 \\
\hline NGC 6981 & 12.8 & $-154_{-13}^{+10}$ & $-4_{-25}^{+14}$ & $230_{-7}^{+10}$ & $22.3_{-1.3}^{+2.1}$ & $0.3_{-0.1}^{+0.3}$ & $0.97_{-0.03}^{+0.01}$ & $108_{-36}^{+-25}$ & 252 & -35 & -89722 \\
\hline NGC 7006 & 38.5 & $-140_{-8}^{+6}$ & $-33_{-7}^{+17}$ & $168_{-12}^{+9}$ & $56.3_{-4.5}^{+2.3}$ & $2.9_{-1.5}^{+0.7}$ & $0.90_{-0.02}^{+0.05}$ & $133_{-13}^{+13}$ & 760 & -1170 & -52073 \\
\hline NGC 7078 & 10.6 & $8_{-12}^{+11}$ & $118_{-11}^{+8}$ & $122_{-10}^{+9}$ & $10.6_{-0.5}^{+0.5}$ & $3.5_{-0.5}^{+0.4}$ & $0.50_{-0.04}^{+0.05}$ & $28_{-1}^{+3}$ & 140 & 1119 & -119947 \\
\hline NGC 7089 & 10.4 & $170_{-6}^{+5}$ & $-18_{-15}^{+12}$ & $243_{-8}^{+8}$ & $18.8_{-1.0}^{+1.7}$ & $0.6_{-0.3}^{+0.2}$ & $0.94_{-0.02}^{+0.03}$ & $119_{-20}^{+1 \frac{1}{2}}$ & 214 & -147 & -97640 \\
\hline NGC 7099 & 7.2 & $-32_{-9}^{+14}$ & $-55_{-12}^{+16}$ & $126_{-9}^{+8}$ & $8.2_{-0.5}^{+0.6}$ & $1.0_{-0.3}^{+0.3}$ & $0.78_{-0.05}^{+0.06}$ & $119_{-6}^{+3}$ & 94 & -234 & -137480 \\
\hline Pal 12 & 15.7 & $146_{-37}^{+22}$ & $304_{-24}^{+\overline{2} \overline{2}}$ & $356_{-17}^{+12}$ & $72.0_{-16.0}^{+18.9}$ & $15.5_{-0.9}^{+1.0}$ & $0.65_{-0.08}^{+0.05}$ & $67_{-2}^{+2}$ & 1178 & 2142 & -41826 \\
\hline Pal 13 & 27.0 & $268_{-11}^{+6}$ & $-78_{-17}^{+21}$ & $289_{-12}^{+9}$ & $87.1_{-13.4}^{+8.6}$ & $8.3_{-1.9}^{+0.9}$ & $0.83_{-0.02}^{+0.03}$ & $115_{-6}^{+8}$ & 1346 & -1586 & -38661 \\
\hline NGC 7492 & 25.4 & $\begin{array}{r}-87_{-22}^{+16} \\
\end{array}$ & $\begin{array}{r}-13_{-7}^{+9} \\
\end{array}$ & $108_{-10}^{+19}$ & $\begin{array}{r}28.2_{-2.3}^{+2.5} \\
\end{array}$ & $\begin{array}{l}3.1_{-1.0}^{+1.4} \\
\end{array}$ & $0.80_{-0.07}^{+0.06}$ & $\begin{array}{r}95_{-5}^{+6} \\
\end{array}$ & 352 & -120 & -77041 \\
\hline
\end{tabular}


Table 2: Globular clusters with modified classification.

\begin{tabular}{|l|r|r||l|r|r||l|r|r|}
\hline Name & GS & GS(m) & Name & GS & GS(m) & Name & GS & GS(m) \\
\hline E 3 & H99 & D & NGC 6254 & LE & D & NGC 6553 & B & D \\
NGC 5466 & Seq & GE & NGC 6304 & B & D & NGC 6569 & B & D \\
NGC 5634 & H99 & GE & Liller 1 & XXX & B & ESO 456-78 & B & D \\
NGC 5694 & HE & GE & NGC 6388 & B & Seq & NGC 6584 & HE & GE \\
NGC 5824 & Sgr & H99 & NGC 6401 & LE & Seq & NGC 6712 & LE & GE \\
NGC 5904 & H99 & GE & NGC 6426 & HE & H99 & NGC 6934 & HE & GE \\
Pal 14 & HE & GE & NGC 6539 & B & D & NGC 6981 & H99 & GE \\
NGC 6144 & LE & Seq & NGC 6540 & B & D & NGC 7006 & Seq & GE \\
NGC 6235 & GE & D & NGC 6544 & LE & GE & Pal 13 & Seq & GE \\
\hline
\end{tabular}

The separation of globular clusters into subsystems of the bulge/bar, thick disk, and halo was performed by us in work Bajkova et al. (2020) using a criterion based on the bimodal distribution of globular clusters over parameter $L_{Z} /$ ecc. The composition of the bulge/bar and thick disk reflects these results. In the redistribution of the remaining globular clusters between the halo subsystems (GE, Seq, Sgr, H99), we took into account the parameters of the orbits. For example, globular clusters with strong radially elongated orbits were assigned to GE. The rest of the rearrangement also took into account the proximity of the orbital shapes.

In Fig. 3 the " $L_{Z^{-}}$Energy", " $L_{Z} / e c c-$ Energy" and "Radial velocity - Rotational velocity" diagrams are presented. These diagrams are given both for classification of GCs by Massari et al. (2019) and for the modified one for comparison. As you can see from Fig. 2, the modified classification looks like a more correct one from the point of view of a greater similarity of the orbits of globular clusters included in their subsystem. From a comparison of the diagrams " $L_{Z}-$ Energy", " $L_{Z} / e c c$ - Energy" and "Radial velocity - Rotational velocity", it can also be concluded that the modified classification is more organic.

\section{Conclusions}

For the first time since the emergence of the Gaia Data Release 2, a catalog of orbits for 152 galactic globular clusters, which form an almost complete known population, is presented.

The main orbital parameters of globular clusters are determined. The astrometric data catalog compiled by Vasiliev (2019) was used as data for calculating the initial 6d phase space (heliocentric positions and velocities of globular clusters) needed for orbit construction. The orbits were integrated for 5 Gyr backward.

For integrating the orbits, we used a recently obtained by Bajkova \& Bobylev (2016) the best-fit model of an axisymmetric Galactic potential with a dark halo in the form of NFW (Navarro et al., 1997) using data on circular velocities of Galactic objects in a wide range of galactocentric distances (data on HI region, masers, and catalog of Bhattacharjee et al. (2014)).

Based on the analysis of the obtained orbits and their properties, we have formed a modified composition of the subsystems of globular clusters, slightly different from the composition presented in Massari et al. (2019). This modification affected 27 GCs. The modified classification looks like a more correct one from the point of view of a greater similarity of the orbits of globular clusters included in their subsystem. 

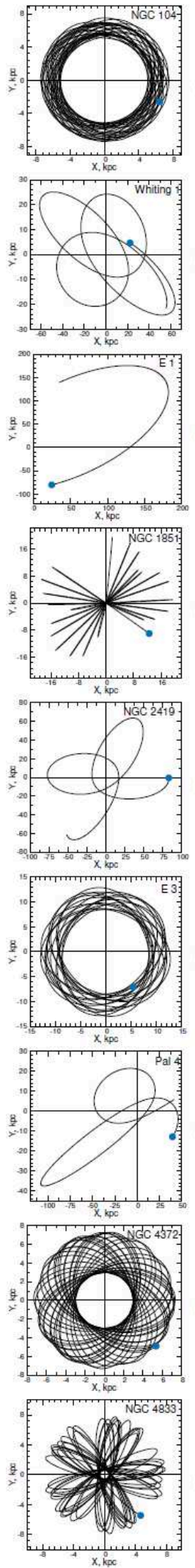
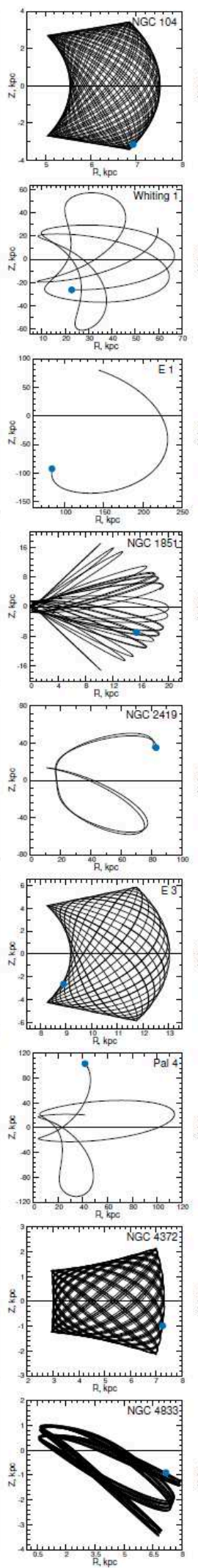
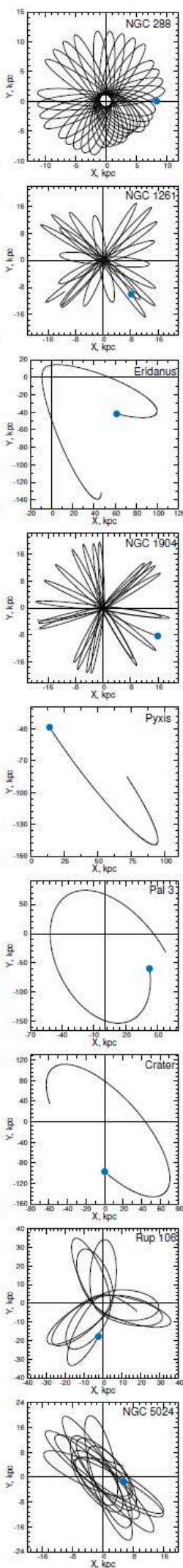
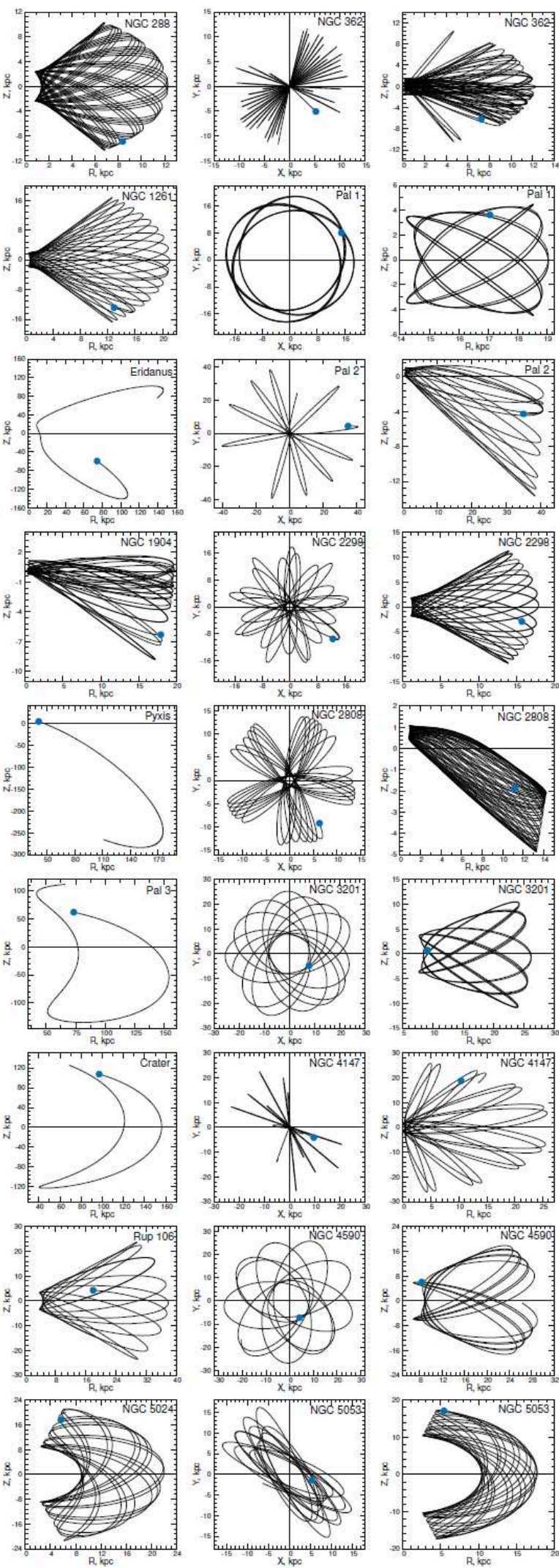

Figure 2: Orbits of the GCs obtained by integrating for 5 Gyr backward. (X,Y) and (R,Z) projections are given. The blue filled circle indicates the beginning of the orbit. 

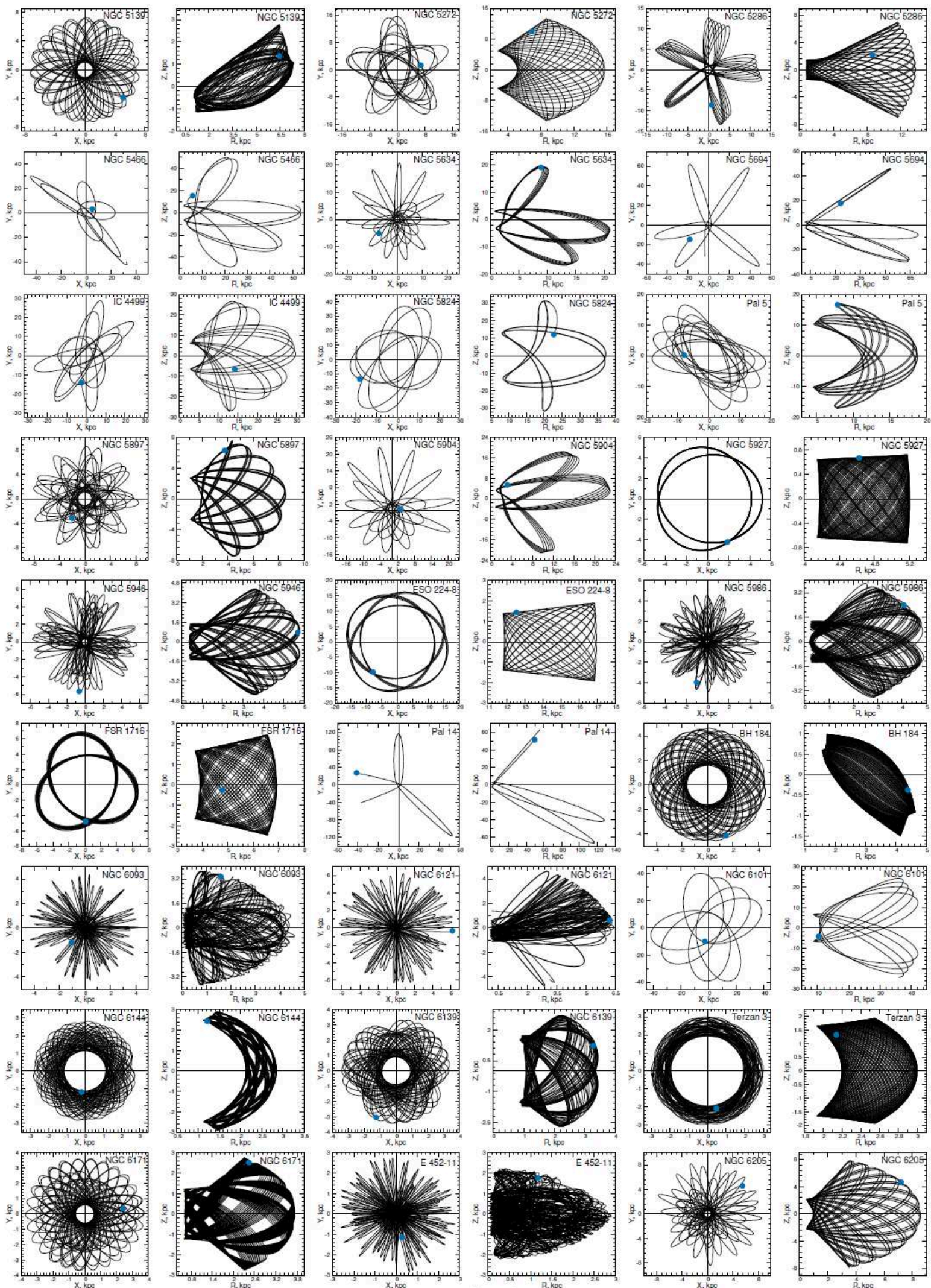

Fig. 2 - continued
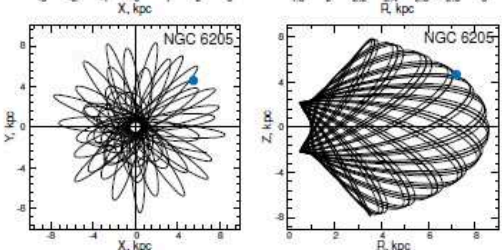

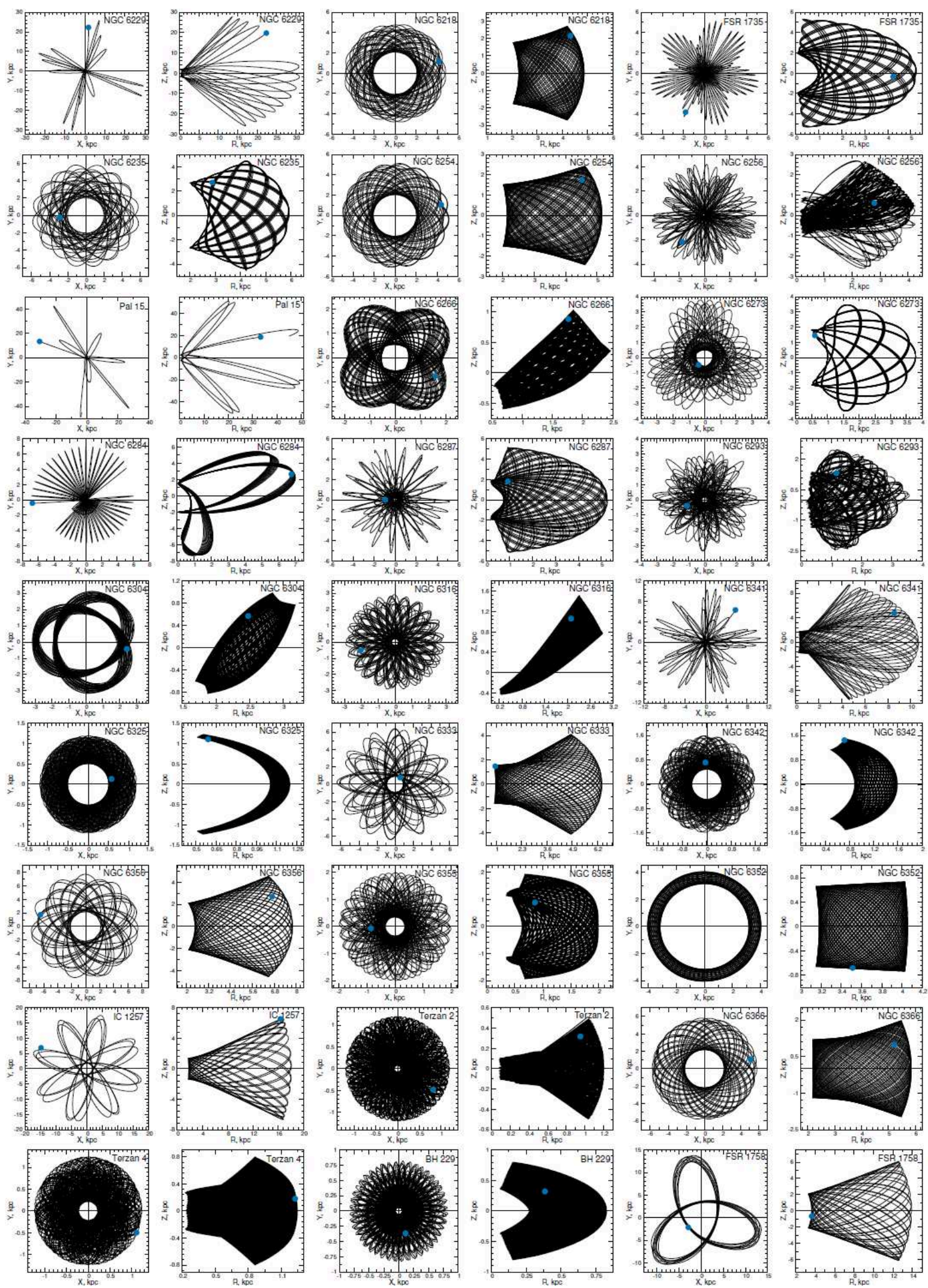

Fig. 2 - continued 

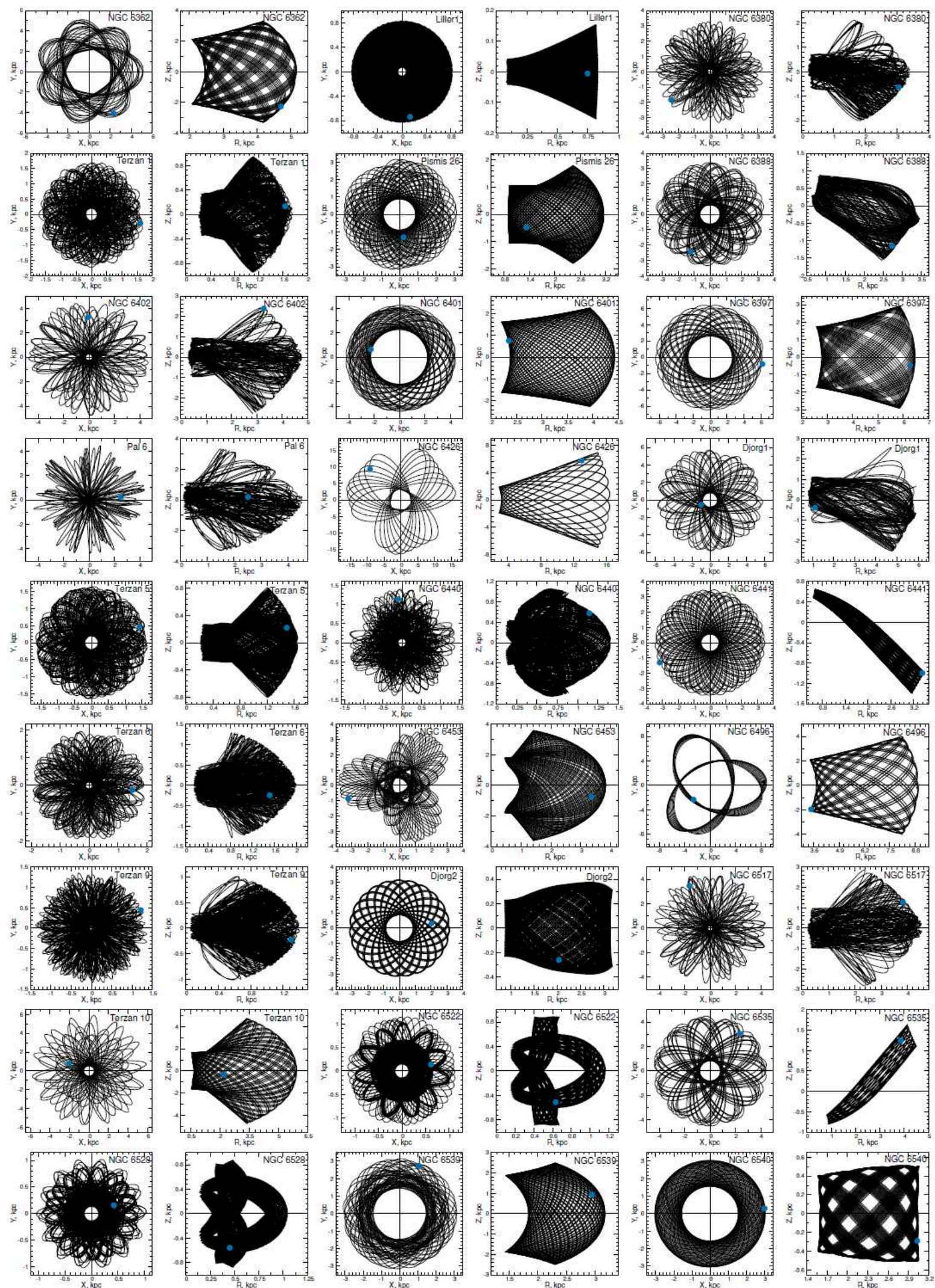

Fig. 2 - continued 

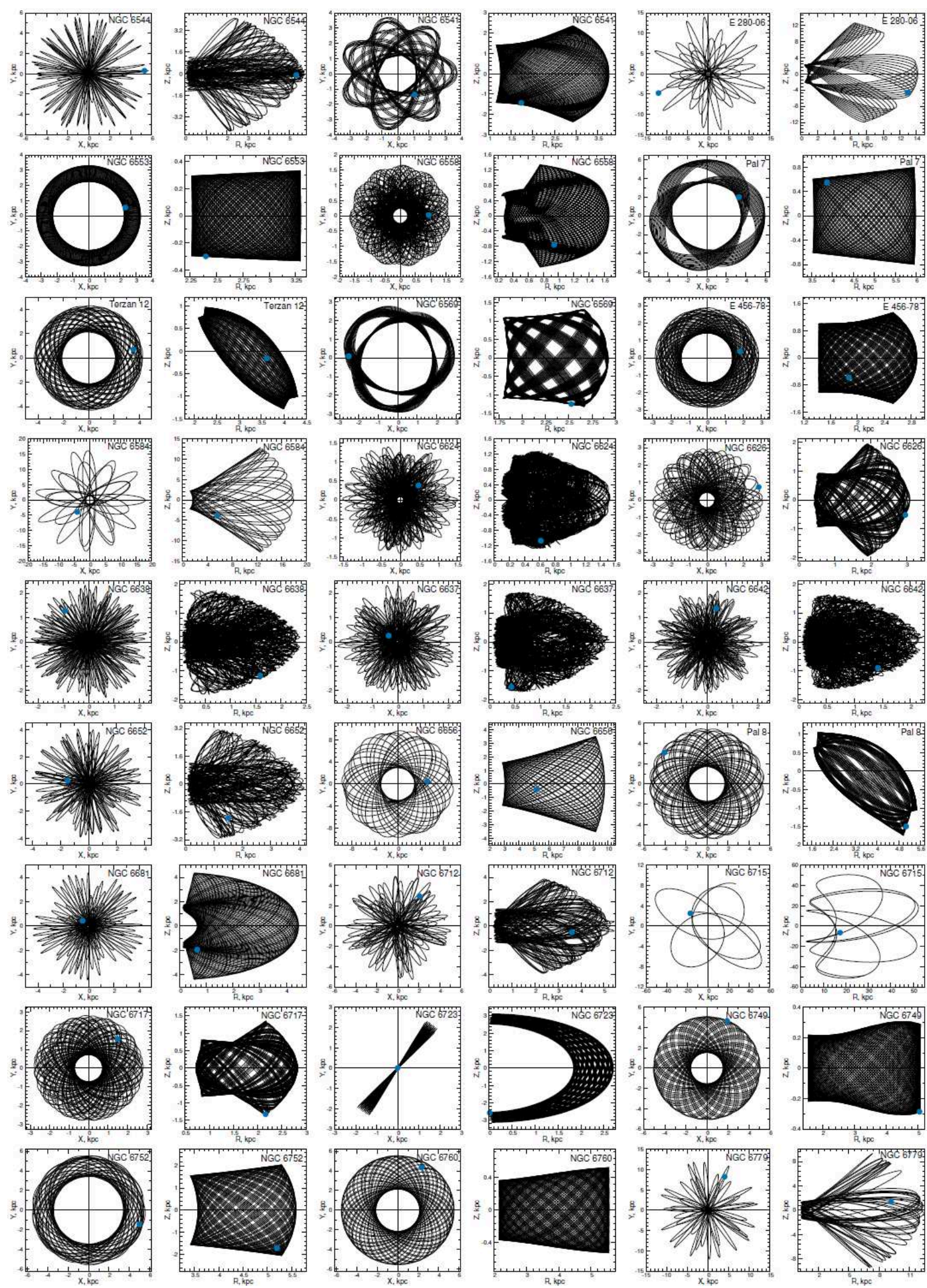

Fig. 2 - continued 

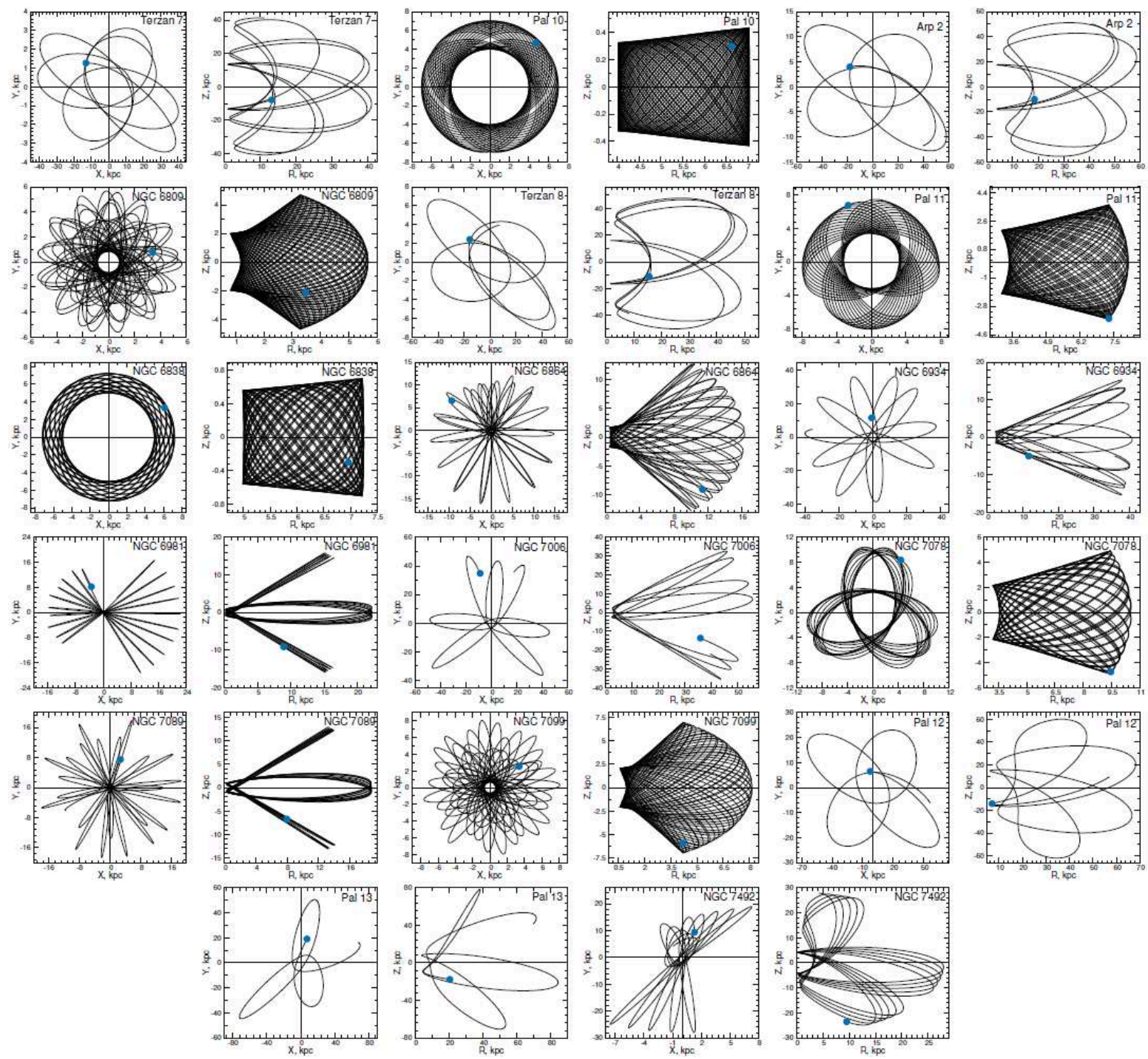

Fig. 2 - continued 

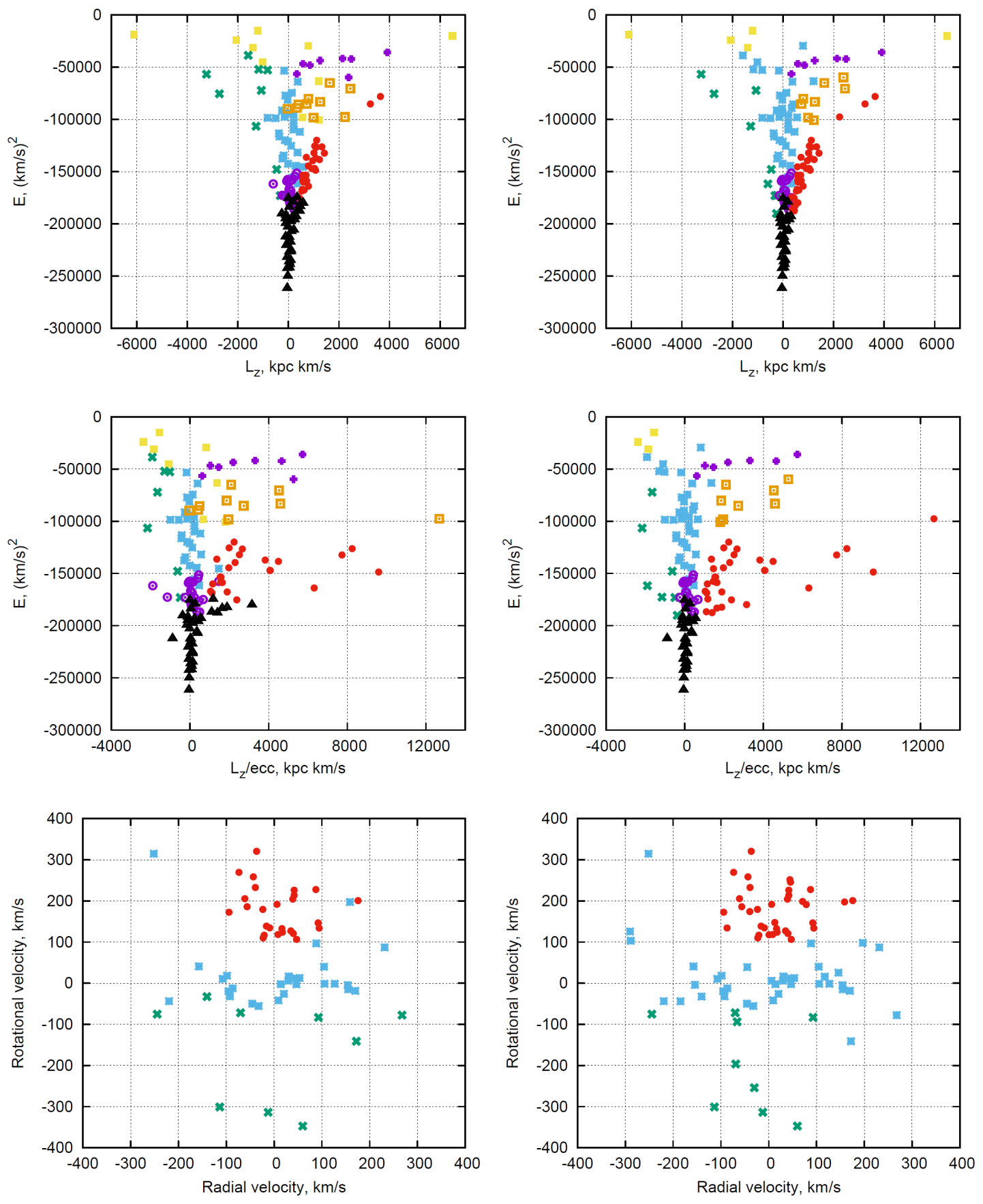

Figure 3: The " $L_{Z}-$ Energy" (top panel), " $L_{Z} /$ ecc - Energy" (middle panel) and "Radial velocity - Rotational velocity" (bottom panel) diagrams for GCs. Classification by Massari et al. (2019) (left-hand panels); the modified classification (right-hand panels). Colour-coded according to their belonging to different subsystems (red symbols mark the disk, black is for the bulge, blue is for GaiaEnceladus (Gaia-Sausage), orange for Helmi Streams, green for Sequoia, violet crosses for Sagittarius, yellow for the high-energy group, violet circles for the low-energy group. For visualisation purposes, clusters E 1 with extremely negative $L_{Z}$ has not been plotted. 


\section{Acknowledgements}

The authors would like to express gratitude to the anonymous referee for useful remarks, the consid-

eration of which made it possible to significantly improve the article. The authors are also grateful to Dr. Dambis A.K. for useful discussion.

\section{References}

- Bajkova, A.T. \& Bobylev, V.V. 2016, Ast. Lett., 42, 567

- Bajkova, A.T. \& Bobylev, V.V. 2017, OAst, 26, 72

- Bajkova, A.T., Carraro, G., Korchagin, V.I., Budanova, N.O. \& Bobylev, V.V. 2020, ApJ, 895, 69

- Baumgardt, H., Hilker, M., Sollima, A. \& Bellini A. 2019, MNRAS, 482, 5138

- Bhattacharjee, P., Chaudhury, S. \& Kundu, S. 2014, ApJ, 785, 63

- Bobylev, V.V. \& Bajkova A.T. 2016, Ast. Lett., 42, 1

- Harris, W. 2010, arXiv: 1012.3224

- Gaia Collaboration, Helmi, A., van Leeuwen, F., McMillan, P.J., et al. 2018, A\&A, 616, 12

- Irrgang, A., Wilcox, B., Tucker, E. \& Schiefelbein, L. 2013, A\&A, 549, 137

- Koppelman, H.H. \& Helmi, A. 2020, arXiv: 2006.16283

- Massari, D., Koppelman, H. H. \& Helmi, A. 2019, A\&A, 630, L4

- Miyamoto, M. \& Nagai, R. 1975, PASJ, 27, 533

- Myeong, G. C., Vasiliev, E., Iorio, G., Evans, N. W. \& Belokurov, V. 2019, MNRAS, 488, 1235

- Navarro, J.F., Frenk, C.S. \& White, S.D.M. 1997, ApJ, 490, 493

- Schonrich, R., Binney, J. \& Dehnen, W. 2010, MNRAS, 403, 1829

- Vasiliev, E. 2019, MNRAS, 484, 2832

- Villanova, S., Monaco, L., Geisler D., et al. 2019, ApJ, 882, 174 